NASA Contractor Report 187610

ICASE Report No. 91-64



ON THE LINEAR STABILITY OF COMPRESSIBLE PLANE COUETTE FLOW

Peter W. Duck

Gordon Erlebacher

M. Yousuff Hussaini

Contract No. NAS1-18605

July 1991

Institute for Computer Applications in Science and Engineering NASA Langley Research Center

Hampton, Virginia 23665-5225

Operated by the Universities Space Research Association

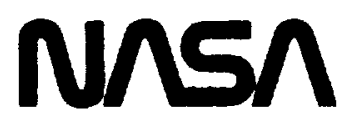

National Aeronautics and

Space Administration

Langley Research Center

Hamplon, Virginia 23665.5225 


\title{
ON THE LINEAR STABILITY OF COMPRESSIBLE PLANE COUETTE FLOW
}

\author{
Peter W. Duck ${ }^{1}$ \\ Department of Mathematics \\ University of Manchester \\ Manchester, M13 9PL, UK \\ Gordon Erlebacher and M. Yousuff Hussaini \\ Institute for Computer Applications in Science and Engineering \\ NASA Langley Research Center \\ Hampton, VA 23665
}

\begin{abstract}
The linear stability of compressible plane Couette flow is investigated. The correct and proper basic velocity and temperature distributions are perturbed by a small amplitude normal mode disturbance. The full small amplitude disturbance equations are solved numerically at finite Reynolds numbers, and the inviscid limit of these equations is then investigated in some detail. It is found that instability can occur, although the stability characteristics of the flow are quite different from unbounded flows. The effects of viscosity are also calculated, asymptotically, and shown to have a stabilizing role in all the cases investigated. Exceptional regimes to the problem occur when the wavespeed of the disturbances approaches the velocity of either of the walls, and these regimes are also analyzed in some detail. Finally, the effect of imposing radiation-type boundary conditions on the upper (moving) wall (in place of impermeability) is investigated, and shown to yield results common to both bounded and unbounded flows.
\end{abstract}

\footnotetext{
${ }^{1}$ This research was supported by the National Aeronautics and Space Administration under NASA Contract No. NAS1-18605 while the authors were in residence at the Institute for Computer Applications in Science and Engineering (ICASE), NASA Langley Research Center, Hampton, VA 23665.
} 



\section{Introduction}

Incompressible plane Couette flow possesses, perhaps, the simplest exact solution of the Navier-Stokes equations, and, (probably as a consequence) the study of the stability of this flow has been the subject of considerable attention over the years. Numerical studies of the linear stability problem have been carried out by Grohne (1954), Gallagher and Mercer (1962, 1964), Deardorff (1963), Davey (1973), and Gallagher (1974); however, all these studies found no evidence of instability. A number of analytic studies have also been carried out on this problem. Wasow (1953) showed that the flow is stable at all streamwise numbers $(\alpha)$ if the Reynolds number (Re) is sufficiently large. The stability of the flow at low Reynolds number was demonstrated by Synge (1938). Dikii (1964) proved that all modes with wavespeeds equal to the average of the wall velocities were stable and indeed that the imaginary component of the wavespeed $\operatorname{Im}\{c\}<-\alpha /$ Re. The first general proof of stability appears to be due to Romanov (1973). He showed that all normal modes of the linear problem are damped for $\alpha \geq 0, \operatorname{Re}>0$. Exact solutions of the Orr-Sommerfeld equations have been obtained by Reid (1979).

Another issue that has been studied is the question of a continuous spectrum. Case $(1960,1961)$ showed that the time dependent inviscid problem has a continuous spectrum which decays in time as $1 / t$, this spectrum arising as a direct consequence of the singularity of the inviscid equations at the critical layer. Shivamoggi (1982) presented an example of a continuous spectrum which decayed as an exponential in time.

A detailed analysis of modes for the large Reynolds number limit for general mean flows is given by Morawetz (1952), which has implications for plane Couette flow. She classified the modes into three sets. In the first set are eigenvalues which approach the inviscid eigenvalues in the limit of infinite Reynolds number. Since there are no discrete inviscid eigenvalues in the case of plane Couette flow, this first set is empty. In the second set, there is an infinity of eigensolutions unrelated to the inviscid problem, satisfying

$$
\left|c-c_{n}\right|<\Delta(\alpha \operatorname{Re})^{-\frac{1}{2}}
$$

where $\Delta$ is a constant, and $c_{n}$ is a root of

$$
\operatorname{Im} \int_{y_{1}}^{y_{2}}\left[i U_{0}(y)-c_{n}\right]^{\frac{1}{2}} d y=\frac{n \pi}{2 \sqrt{\alpha \mathrm{Re}}}
$$

where $n$ is any integer, $U_{0}(y)$ is the velocity profile, $\alpha$ is the wavenumber, and the flow extends from $y=y_{1}$ to $y=y_{2}$. These modes are always stable (if Re is sufficiently large). In the third class are eigensolutions of the viscous problem that, as $\alpha \operatorname{Re} \rightarrow \infty$, approach a finite V-shaped strip in c-space defined by one branch of

$$
R l \int_{y_{c}}^{y_{1}}\left[i\left(U_{0}(y)-c\right)\right]^{\frac{1}{2}} d y=0
$$

and one branch of

$$
R l \int_{y_{c}}^{y_{2}}\left[i\left(U_{0}(y)-c\right)\right]^{\frac{1}{2}} d y=0
$$

where $U_{0}\left(y_{c}\right)=R l\{c\}$. These modes are either neutral or stable. 
However, there does exist a dichotomy between the theoretical and computational results described above and experimental results at large Reynolds numbers, in which instabilities are certainly observed (Taylor 1936, Reichardt 1956, Robertson 1959). A number of attempts have been made to explain this through the inclusion of nonlinear terms. Investigations of this type include the work of Watson (1960), Eckhaus (1965), Hains (1967), Kuwahara (1967), Reynolds and Potter (1967), Ellingsen, Gjevik, and Palm (1970), and Lessen and Cheifetz (1975). These studies used a variety of techniques, including varying degrees of mathematical rigor, and led to a number of conclusions (some partly contradictory), although on balance, the evidence was that finite amplitude effects could, indeed, destabilize the flow. Additionally, Romanov (1973) considered the nonlinear initial-value problem and showed that there is a unique solution which is asymptotically stable if the norm of the initial disturbances in Sobolev space is sufficiently small.

The linear stability of compressible flows is considerably less well understood than corresponding incompressible flows. Most of the work (which has for the most part been based on the parallel mean flow assumption) has been with regard to boundary-layer flows (e.g., Lees and Lin 1946, Mack 1963, 1965a,b, 1969, 1984, 1987, 1990) and more recently to jets and shear layers (e.g., Tam and Hu 1989a,b, Greenough et al. 1988, Papageorgiou 1990, Jackson and Grosch 1989, Mack 1990).

The first (of a number) of distinguishing features of the stability of compressible flows was found by Lees and Lin (1946). This relates to the replacement of the (classical) inflection point condition in the streamwise velocity profile (for the existence of neutral inviscid modes), by the generalized inflection point (G.I.P.) condition which involves the mean density distribution also. This condition relates to the existence of neutral "inviscid" modes (i.e., modes of wavelength comparable to the characteristic scales of the mean flow, for example the displacement thickness in the case of boundary-layer flows, or lateral extent in the case of confined flows).

Another effect of compressibility is the frequent existence of multiple modes of instability in supersonic flows. In addition to the modes related to the G.I.P., a further class of modes may exist; these are linked to the so-called supersonic neutral modes (as opposed to subsonic neutral modes associated with G.I.P.'s). Such neutral modes are readily distinguishable, because of the oscillatory nature of the eigensolutions in the free stream, while subsonic neutral modes exhibit exponential decay of their eigensolutions in the free stream. Mack $(1963,1965 a, b, 1969,1984,1987,1990)$ has shown how such modes are extremely important in compressible boundary-layer flows, particularly in the case of boundary layers on cooled walls, where these modes are often associated with the largest amplification rates. Such modes have also been shown to exist and be important in other classes of flow by Tam and Hu (1989), Greenough (1989), Zhuang, Kubota, and Dimotakis (1990) (confined twodimensional supersonic mixing layers), Macaraeg (1990), and by Jackson and Grosch (1989) (compressible mixing layers). However, to date, a simple mechanism that explains why and precisely under what conditions these types of mode exist has yet to be propounded. Some general remarks concerning stability at large Reynolds numbers have been made, however, by Morawetz (1959), in particular, the presence of inner viscous regions and their nature was vigorously established in both the incompressible and compressible cases. In the case 
of stable disturbances, with finite damping, it was shown that a finite viscous region in the interior of the fluid, no matter how large the Reynolds number, exists. In the case of unstable disturbances, with finite amplification, there is no inner viscous region if the Reynolds number of the mean flow is large enough. In the case of neutral disturbances (for constant Prandtl number) it was also rigorously shown that if a viscous region does exist, its width shrinks to zero with an increase in Reynolds number.

The object of this study is to analyze some of the characteristics of compressible plane Couette flow. Although analytic expressions for the mean streamwise velocity $U_{0}(y)$ and temperature $T_{0}(y)$ are not available for general viscosity laws, $T_{0}(y)$ may be expressed as a second-order polynomial in $U_{0}(y)$, and hence it is relatively straightforward to generate mean flow profiles, under different conditions. Some work has previously been carried out investigating the compressible stability of plane Couette flow. Glatzel (1989) has considered this problem, and has carefully studied the least stable eigenmodes. He also derived critical Reynolds numbers for the instabilities that were found. However, this work is for the special case of constant viscosities, density and pressure (implying constant temperature also), which lead to a fourth-order system (rather than a sixth-order system). Girard (1988) considered the same problem, and also assumed constant viscosities (although he did allow for variations in density and temperature); instabilities were again found to occur. In this paper, we choose to consider the stability of the mean flow profile described above, which is a correct and proper solution of the full compressible Navier-Stokes equations. We show that the details of the mean flow profile have a profound effect on the stability of the flow.

The layout of the paper is as follows. In Section 2 we formulate the problem, stating our fundamental assumptions and equations of motion. In Section 3 we derive the equations of motion for the basic flow, in which the problem is reduced to that of a straightforward, although nonlinear, first order system that may be solved by means of standard numerical means. In Section 4 we derive the (full) small amplitude disturbance equations, neglecting only terms in perturbation amplitude squared, and we describe a numerical scheme to treat this system, together with a number of numerical results. In Section 5 we consider the inviscid limit of these equations; we show how the so-called "generalized inflection point" is relevant in this context, and under what circumstances we can expect such a point to occur with our basic profile.

Numerical results for the inviscid problem are presented in Section 6, and, most importantly, it is shown that unstable modes are possible, although according to inviscid theory, there are many regions where many modes are neutrally stable. These neutral modes are investigated further in Section 7, in which the effects of viscosity are included, and shown to always have a stabilizing role for high Reynolds numbers. However, this study also raises important questions regarding the applicability of our results, in particular in regions of changeover from the neutral to non-neutral state. This region is investigated in some detail in Section 8. Because of the apparent discrepancy in results between bounded and unbounded stability analyses, in Section 9 we consider a change in boundary conditions on the upper (moving) wall, from one of impermeability to one of radiation. Finally, in Section 10 we present some conclusions. 


\section{Formulation}

We assume that we have a compressible, Newtonian, perfect fluid between two infinite parallel planes defined by $y^{*}=0$ and $y^{*}=h$. The $x^{*}$ axis is taken to lie in the plane of the lower wall. We take the fluid to have density $\rho^{*}$, viscosity $\mu^{*}$, second coefficient of viscosity $\zeta^{*}$ (which may be taken to be zero for a monotonic gas). The upper wall has velocity $U_{\infty}$ (a subscript $\infty$ refers to unperturbed conditions on the upper wall), taken parallel to the plane of the wall, while the lower wall is at rest. Although the basic flow will be taken to be one-dimensional, later we shall consider two-dimensional perturbations of this flow. The velocity components are taken to be $\mathbf{u}^{*}=\left(u^{*}, v^{*}\right)$ in the $x^{*}$ and $y^{*}$ directions respectively, and the pressure and temperature are written as $p^{*}$ and $T^{*}$ respectively. We non-dimensionalize velocities with respect to $U_{\infty}$, lengthscales with respect to $h$, density with respect to $\rho_{\infty}^{*}$, viscosities with respect to $\mu_{\infty}^{*}$, temperature with respect to $T_{\infty}^{*}$ and pressure with respect to $\rho_{\infty}^{*} R^{*} T_{\infty}^{*}$, where the gas constant $R^{*}=C_{p}-C_{v}$, and $C_{p}$ and $C_{v}$ are the specific heats at constant pressure and volume respectively; non-dimensional quantities are denoted using the same notation as corresponding dimensional quantities, except without the superscript asterisk.

The continuity equation may then be written

$$
\frac{\partial \rho}{\partial t}+\frac{\partial}{\partial x}(\rho u)+\frac{\partial}{\partial y}(\rho v)=0 .
$$

Here, and throughout the paper, we assume that the appropriate dimensional timescale is $O\left(h / U_{\infty}\right)$.

The momentum equations are written

$$
\begin{aligned}
& \rho\left[\frac{\partial u}{\partial t}+u \frac{\partial u}{\partial x}+v \frac{\partial u}{\partial y}\right]=-\frac{1}{\gamma M_{\infty}^{2}} \frac{\partial p}{\partial x} \\
& +\frac{1}{\operatorname{Re}}\left\{\frac{\partial}{\partial x}\left[2 \mu \frac{\partial u}{\partial x}+\lambda \nabla \cdot \mathbf{u}\right]+\frac{\partial}{\partial y}\left[\mu\left(\frac{\partial u}{\partial y}+\frac{\partial v}{\partial x}\right)\right]\right\}, \\
& \rho\left[\frac{\partial v}{\partial t}+u \frac{\partial v}{\partial x}+v \frac{\partial v}{\partial y}\right]=-\frac{1}{\gamma M_{\infty}^{2}} \frac{\partial p}{\partial y} \\
& +\frac{1}{\operatorname{Re}}\left\{\frac{\partial}{\partial y}\left[2 \mu \frac{\partial v}{\partial y}+\lambda \nabla \cdot \mathbf{u}\right]+\frac{\partial}{\partial x}\left[\mu\left(\frac{\partial u}{\partial y}+\frac{\partial v}{\partial x}\right)\right]\right\} .
\end{aligned}
$$

Here the Reynolds number Re is defined by

$$
\operatorname{Re}=\frac{U_{\infty} \rho_{\infty}^{*} h}{\mu_{\infty}^{*}},
$$

the Mach number $M_{\infty}$ by

$$
M_{\infty}=U_{\infty} / \sqrt{\gamma R^{*} T_{\infty}^{*}},
$$

and the ratio of specific heats by $\gamma$. 
The non-dimensional form of the energy equation used is

$$
\begin{gathered}
\rho\left[\frac{\partial T}{\partial t}+u \frac{\partial T}{\partial x}+v \frac{\partial T}{\partial y}\right]-\left(\frac{\gamma-1}{\gamma}\right)\left[\frac{\partial p}{\partial t}+u \frac{\partial p}{\partial x}+v \frac{\partial p}{\partial y}\right]=\frac{1}{\operatorname{Re}}\left\{\frac{\partial}{\partial y}\left[\frac{\mu}{\sigma} \frac{\partial T}{\partial y}\right]+\frac{\partial}{\partial x}\left[\frac{\mu}{\sigma} \frac{\partial T}{\partial x}\right]\right\} \\
+\frac{2 \mu(\gamma-1) M_{\infty}^{2}}{\operatorname{Re}}\left[\left(\frac{\partial u}{\partial x}\right)^{2}+\left(\frac{\partial v}{\partial y}\right)^{2}+\frac{1}{2}\left(\frac{\partial u}{\partial y}+\frac{\partial v}{\partial x}\right)^{2}+\frac{\lambda}{2 \mu}\left(\frac{\partial u}{\partial x}+\frac{\partial v}{\partial y}\right)^{2}\right]
\end{gathered}
$$

Here $\sigma$ is the Prandtl number

$$
\sigma=\mu^{*} C_{p} / K^{*},
$$

where $K^{*}$ is the coefficient of heat conductivity. The equation of state is simply

$$
p=\rho T .
$$

We assume that viscosity depends solely on temperature, and in particular we assume Sutherland's law

$$
\mu=T^{3 / 2}\left(\frac{1+C}{T+C}\right),
$$

where $C$ is a constant. Finally, $\lambda=\zeta-2 / 3 \mu$ and the Stokes assumption $\zeta=0$ is assumed throughout the paper.

In the following section we consider the basic flow which we expect to depend on $y$ only.

\section{Compressible Couette Flow}

We seek a solution of (2.1) - (2.6) which is dependent on $y$ only, together with a constant mean pressure. By continuity we must have $v=0$. We then seek a solution of the form

$$
u=U_{0}(y), T=T_{0}(y), \mu=\mu_{0}(y) .
$$

We then have

$$
\begin{gathered}
\left(\mu_{0} U_{0 y}\right)_{y}=0 \\
{\left[\frac{\mu_{0} T_{0 y}}{\sigma}\right]_{y}+(\gamma-1) M_{\infty}^{2} \mu_{0}\left(U_{0 y}\right)^{2}=0}
\end{gathered}
$$

subject to

$$
\begin{array}{ll}
U_{0}(0)=0, & U_{0}(1)=1 \\
T_{0}(0)=T_{w}, & T_{0}(1)=1 .
\end{array}
$$

It follows immediately from (3.2) that the shear stress $\tau$ is a constant through the profile, i.e.,

$$
\tau=\mu_{0} U_{0 y}=\text { constant. }
$$

The energy equation may then be written as

$$
\left[\frac{\mu_{0}}{\sigma} T_{0 y}+(\gamma-1) M_{\infty}^{2} U_{0} \tau\right]_{y}=0 .
$$


Defining the recovery temperature (i.e., the wall temperature with adiabatic conditions) as

$$
T_{r}=1+\frac{(\gamma-1)}{2} \sigma M_{\infty}^{2}
$$

and the recovery factor

$$
r=\frac{T_{w}}{T_{r}}
$$

(3.6) may be integrated and written in the form

$$
T_{0}=T_{r}\left\{r+(1-r) U_{0}-\left(1-\frac{1}{T_{r}}\right) U_{0}^{2}\right\} .
$$

Equations (3.5) and (3.9), together with an appropriate viscosity law (we used (2.9)) and boundary conditions (3.4) then completely determine the problem; $\tau$ is unknown a priori, and must therefore be determined as part of an iterative process. The mean pressure profile is constant.

\section{The Small-Amplitude Disturbance Equations}

Here we take the solution to be that of Couette flow (as discussed in the previous section) together with a small amplitude perturbation. More specifically, we write

$$
\begin{aligned}
u & =U_{0}(y)+\delta \tilde{u}(y) E+O\left(\delta^{2}\right) \\
v & =\delta \alpha \tilde{v}(y) E+O\left(\delta^{2}\right) \\
p & =1+\delta \tilde{p}(y) E+O\left(\delta^{2}\right) \\
\rho & =\rho_{0}(y)+\delta \tilde{\rho}(y) E+O\left(\delta^{2}\right), \\
\mu & =\mu_{0}(y)+\delta \tilde{\mu}(y) E+O\left(\delta^{2}\right) \\
\zeta & =\zeta_{0}(y)+\delta \tilde{\zeta}(y) E+O\left(\delta^{2}\right), \\
T & =T_{0}(y)+\delta \tilde{T}(y) E+O\left(\delta^{2}\right)
\end{aligned}
$$

where

$$
E=\exp [i \alpha(x-c t)]
$$

and $\delta$ is the (small) amplitude of the perturbation. If we take terms $O(\delta)$ in $(2.1)-(2.3)$, (2.6) we obtain the following full, small disturbance equations:

$$
\begin{aligned}
& -i c \tilde{\rho}+i \rho_{0} \tilde{u}+i U_{0} \tilde{\rho}+\tilde{v} \rho_{0 y}+\rho_{0} \tilde{v}_{y}=0 \\
& \rho_{0}\left[-i \alpha c \tilde{u}+i \alpha U_{0} \tilde{u}+\alpha \tilde{v} U_{0 y}\right]+\frac{i \alpha}{\gamma M_{\infty}^{2}} \tilde{p}= \\
& \frac{1}{\operatorname{Re}}\left\{-2 \mu_{0} \alpha^{2} \tilde{u}+\lambda_{0}\left[-\alpha^{2} \tilde{u}+i \alpha \tilde{v}_{y}\right]+\mu_{0 y}\left[\tilde{u}_{y}+i \alpha \tilde{v}\right]+\mu_{0}\left[\tilde{u}_{y y}+i \alpha \tilde{v}_{y}\right]+\tilde{\mu}_{y} U_{0 y}+\tilde{\mu} U_{0 y y}\right\} \\
& \rho_{0}\left[-i \alpha^{2} \tilde{v}+i \alpha^{2} U_{0} \tilde{v}\right]+\frac{\tilde{p}_{y}}{\gamma M_{\infty}^{2}}= \\
& \frac{1}{\operatorname{Re}}\left\{2 \mu_{0 y} \tilde{v}_{y}+2 \mu_{0} \tilde{v}_{y y}+\lambda_{0 y}\left(i \alpha \tilde{u}+\tilde{v}_{y}\right)+\lambda_{0}\left(i \alpha \tilde{u}_{y}+\tilde{v}_{y y}\right)+\mu_{0}\left(i \alpha \tilde{u}_{y}-\alpha^{2} \tilde{v}\right)+i \alpha \tilde{\mu} U_{0 y}\right\}
\end{aligned}
$$




$$
\begin{aligned}
& \rho_{0}\left[-i \alpha c \tilde{T}+i \alpha U_{0} \tilde{T}+\alpha \tilde{v} T_{0 y}\right]-\left(\frac{\gamma-1}{\gamma}\right)\left[-i \alpha c \tilde{p}+i \alpha U_{0} \tilde{p}\right]= \\
& \quad \frac{1}{\sigma \operatorname{Re}}\left\{\mu_{0} \tilde{T}_{y y}+\mu_{0 y} \tilde{T}_{y}+\tilde{\mu} T_{0 y y}+\tilde{\mu}_{y} T_{0 y}-\alpha^{2} \mu_{0} \tilde{T}\right\} \\
& +\frac{(\gamma-1) M_{\infty}^{2}}{\operatorname{Re}}\left\{\mu_{0} U_{0 y} \tilde{u}_{y}+\tilde{\mu}\left(U_{0 y}\right)^{2}+2 \mu_{0} i \alpha U_{0 y} \tilde{v}\right\} .
\end{aligned}
$$

The perturbation equation of state is then

$$
\tilde{p}=T_{0} \tilde{\rho}+\frac{\tilde{T}}{T_{0}} .
$$

No-slip and impermeability conditions are applied to the velocity perturbations at both walls, namely

$$
\tilde{u}(0)=\tilde{u}(1)=\tilde{v}(0)=\tilde{v}(1)=0 .
$$

The temperature boundary conditions are $\tilde{T}(1)=0$, while on the lower wall,

$$
\tilde{T}(0)=0
$$

for a heated/cooled surface and

$$
\frac{\partial \tilde{T}}{\partial y}=0 \quad \text { on } y=0
$$

for an insulated wall.

We now present results based on the full set of viscous compressible linearized equations, i.e. (4.3)-(4.7) above.

The stability results are obtained from a spectral temporal linear stability code written by Herbert (1990). The code assumes a global representation of all variables in appropriate basis functions which can vary from variable to variable. The basis functions for the velocity components satisfy the imposed boundary conditions. Velocity perturbations are set to zero at $y=0$ and $y=1$. Thus, the velocity basis functions are linear combinations of Chebyshev polynomials. In terms of the Chebyshev polynomials $T_{n}(y)$, these basis functions are defined as

$$
U_{n}(y)=T_{n+2}(y)-T_{n}(y) .
$$

Boundary conditions for the density and temperature perturbations waves are of more general nature, so these variables are represented as a standard Chebyshev series. In this section, only adiabatic results are shown. Thus, the $y$ derivative of the temperature perturbation is zero at the lower wall. The upper wall is insulated; therefore the temperature perturbation is set to zero. The density at the walls is obtained by integration of the continuity equation.

The numerical algorithm departs from standard collocation schemes which operate in physical space. Taking the perturbation density $(\tilde{\rho})$ as an example, let

$$
\tilde{\rho}(y)=\sum_{n} a_{n} T_{n}(y)
$$


and its first derivative

$$
\frac{d \tilde{\rho}(y)}{d y}=\sum_{n} a_{n} \frac{d T_{n}(y)}{d y}
$$

be evaluated at each collocation point. Inserting $\tilde{\rho}$ and the other variables into the linearized stability equations leads to a system of linear equations for the coefficients of the basis functions. From these, one can either calculate a global spectrum using routines from the IMSL library or obtain a single eigenvalue at a sequence of parameter values using a continuation technique. Details are given in Herbert (1990).

Figures 1-5 all correspond to $M_{\infty}=2$. The dependence of the eigenvalues of inviscid character is shown in Figures 1 a-c which correspond to $\alpha=0.05,0.10$ and 0.20 respectively, all at $\operatorname{Re}=2 \times 10^{5}$, and obtained with 100 collocation points. It is clear from the sequence of figures that one inviscid eigenvalue is approximately stationary, while the remaining inviscid eigenvalues originate from very large values of $R l\{c\}$ at very low $\alpha$. A magnification of figure 1c (shown in figure 1d) shows the region of the spectrum corresponding to $0<R l\{c\}<1$. One notes the general "Y"shape of the spectrum as discussed by Morawetz (1952). The spectrum is composed of three "Y" shaped pieces. These are due to the structure of the viscous terms of the two momentum equations and the temperature equation. The coupling between the equations and the non unit Prandtl number leads to the non superposition of the "Y" singular curves as $\operatorname{Re} \rightarrow \infty$. Before drawing any general conclusions however, we show spectra at $\operatorname{Re}=7 \times 10^{5}$ in Figure 2, leaving all other parameters unchanged with respect to Figure 1. As expected, higher resolution is required to properly resolve the spectrum. The sequence of Figures 2a-d correspond to resolutions of 75,100,125 and 150 collocation points respectively. Although Morawetz (1952) predicts that there is a set of "viscous" eigenvalues which lie on the edges of the "V" part of the "Y", packed with a density proportional to $\sqrt{\alpha R e}$, it is clear that at $M_{\infty}=2$, the triple point of the "Y" is cut off by two horizontal bands of eigenvalues. The word horizontal is used here as a qualitative description. At the lower resolution of $N=75$, the bands are slightly wider then for higher $N$. It is not completely clear whether these bands will remain or disappear as $N \rightarrow \infty$. A definitive answer would require further investigation into the properties of the viscous component of the spectrum at high Reynods numbers and high Mach numbers. Comparing Figures $2 \mathrm{~b}$ and $2 \mathrm{~d}$, it is clear that one effect of insufficient resolution is the splitting of the spectrum from the vertical part of the "Y" (which is indicated by "|"). However, such a splitting is not always indicative of loss of resolution (see Figure 5 at $\alpha=0.1, M_{\infty}=5, \operatorname{Re}=2 \times 10^{6}$ ). At $\alpha=3.5, \operatorname{Re}=7 \times 10^{5}$, the horizontal bands become more evident (Figure 3 ). Note that the density of eigenvalues along this band has increased significantly. Figure 4 shows a blow up of the upper left hand corner of the stable diagram. The scales along $R l\{c\}$ and $\operatorname{Im}\{c\}$ are identical to bring out the real shape of one of the "triple points". At $N=150$, one notices the left branch of the "Y" which is at the theoretical angle of $60^{\circ}$. As $N$ changes from 125 to 150 nodes, the horizontal bands shift slightly in the direction of increased stability. Thus, the final converged state of these bands is inconclusive for the chosen set of parameters.

We now turn our attention to a similar set of spectra at $M_{\infty}=5$ which seem to exhibit a more complex character than their $M_{\infty}=2$ counterparts. At $\operatorname{Re}=2 \times 10^{6}, \alpha=0.1$, the three "Y" shaped curves are clearly present. These structures subsist in an unmodified form 
(i.e., without the horizontal bands) at higher Reynolds numbers at the higher $M_{\infty}$. One "Y" is approximately symmetrical about the $R l\{c\}=0.5$ axis. The "l" structure has split into three pieces, one along $R l\{c\}=0.5$, and the other two curves placed symmetrically about $R l\{c\}=0.5$. The actual locus of points along these curves shows a similarity with the continuous temporal boundary-layer spectra discussed in Ashpis and Erlebacher (1989). As the Reynolds number is increased to $5 \times 10^{6}$, the resolution requirements simultaneously increase. Only when the vertical locus of eigenvalues along $R l\{c\}=0.5$ appears, is the resolution adequate, at least for the part of the spectra above it, although this statement is not quite true near the horizontal bands. The unchanging vertical position of these bands as $N$ increases from 125 to 150 indicates that its presence is not an artifact of a loss of resolution.

To complete the picture, Figures 7 and 8 show the spectra at $M_{\infty}=5, \alpha=3.5$ at $\operatorname{Re}=2 \times 10^{5}$ and $\operatorname{Re}=7 \times 10^{5}$. In both cases some inviscid modes (those near the $\operatorname{Im}\{c\}$ axis) have moved into the $0<R l\{c\}<1$ range. As each mode crosses $R l\{c\}=0$ or $R l\{c\}=1$ regions, a critical layer develops; this is examined in Section 6 . Once again, as the Reynolds number increases, the width of the horizontal bands increases, and resolution studies indicate that they do not disappear as $N \rightarrow \infty$.

In anticipation of the results presented in the following sections, we plot the evolution of the mode I phase velocity (finite $R l\{c\}$ as $\alpha \rightarrow 0$ ) as a function of $\alpha$. This is carried out for several resolutions and at two different Reynolds numbers. Although inviscid theory suggests stronger instabilities at $M=5$, viscous calculations indicate that the resolution requirements become much more severe. We therefore restrict ourselves to $M_{\infty}=2$. Both at $\operatorname{Re}=7 \times 10^{5}$ and $\operatorname{Re}=1.4 \times 10^{6} . \operatorname{Im}\{c\}$ exhibits a local maximum near $\alpha=4$. This mode becomes less stable at higher Re, while $\alpha$ shifts to a slightly lower value. As expected, a higher resolution is required at $\operatorname{Re}=1.4 \times 10^{6}(N=150)$, as opposed to $N=100$ at $\operatorname{Re}=7 \times 10^{5}$. Unfortunately, we are not able to increase Re beyond $1.4 \times 10^{6}$ and still obtain accurate results at resolutions not exceeding $N=150$. Figures 9 and 10 show the variation of $R l c_{i}$ and $R l c_{r}$ for both Reynolds numbers. The phase velocity has been scaled by $\sqrt{R e}$ to bring out the one-half power scaling law. Further discussion of Figure 9 is deferred until the asymptotic theory at large Re has been presented.

In the following several sections, we examine solutions of the stability problem in the limit $\operatorname{Re} \rightarrow \infty$. Guided by a number of previous viscous theories, together with the work of Morawetz $(1952,1959)$, we expect, in general, the solution to develop in the form

$$
\begin{aligned}
& \tilde{u}=\tilde{u}_{0}(y)+\operatorname{Re}^{-\frac{1}{2}} \tilde{u}_{1}(y)+O\left(\operatorname{Re}^{-1}\right), \\
& \tilde{v}=\tilde{v}_{0}(y)+\operatorname{Re}^{-\frac{1}{2}} \tilde{v}_{1}(y)+O\left(\operatorname{Re}^{-1}\right), \\
& \tilde{T}=\tilde{T}_{0}(y)+\operatorname{Re}^{-\frac{1}{2}} \tilde{T}_{1}(y)+O\left(\operatorname{Re}^{-1}\right), \\
& \tilde{p}=\tilde{P}_{0}(y)+\operatorname{Re}^{-\frac{1}{2}} \tilde{P}_{1}(y)+O\left(\operatorname{Re}^{-1}\right), \\
& c=c_{0}+\operatorname{Re}^{-\frac{1}{2}} c_{1}+O\left(\operatorname{Re}^{-1}\right) .
\end{aligned}
$$




\section{Inviscid Disturbance Equations}

Taking equations (4.3) - (4.7), and then retaining lowest order terms in Re (see Mack 1984, for example), we obtain the following two first-order equations for the leading order normal velocity $\left(\tilde{v}=\tilde{v}_{0}\right)$ and pressure $\left(\tilde{p}=\tilde{p}_{0}\right)$ perturbations respectively

$$
\begin{gathered}
\tilde{v}_{0 y}-\frac{U_{0 y} \tilde{v}_{0}}{U_{0}-c_{0}}=\frac{i \tilde{p}_{0}}{\gamma M_{\infty}^{2}} \frac{\left\{T_{0}-M_{\infty}^{2}\left(U_{0}-c_{0}\right)^{2}\right\}}{U_{0}-c_{0}} \\
\frac{\tilde{p}_{0 y}}{\gamma M_{\infty}^{2}}=-\frac{i \alpha^{2}}{T_{0}}\left(U_{0}-c_{0}\right) \tilde{v}_{0}
\end{gathered}
$$

where the appropriate boundary conditions to be applied to this system are

$$
\tilde{v}_{0}(0)=\tilde{v}_{0}(1)=0
$$

implying

$$
\tilde{p}_{0 y}(0)=\tilde{p}_{0 y}(1)=0,
$$

where $c_{0}$ is the complex wave-speed of this system. Equations (5.1) and (5.2) may be combined to yield the single second order equation (see for example Mack 1984, 1987)

$$
\frac{d}{d y}\left\{\frac{\left(U_{0}-c_{0}\right) \tilde{v}_{0 y}-U_{0 y} \tilde{v}_{0}}{T_{0}-M_{\infty}^{2}\left(U_{0}-c_{0}\right)^{2}}\right\}=\alpha^{2} \frac{\left(U_{0}-c_{0}\right)}{T_{0}} \tilde{v}_{0}
$$

for $\tilde{v}_{0}$.

Before investigating any of the above systems numerically, it is interesting to study the significance of so-called "generalized inflection points" which are found to be highly significant in the context of compressible flows. If we multiply (5.5) by $\tilde{v}_{0}^{*}$ (the complex conjugate of $\tilde{v}_{0}$ ) and then subtract from the resulting equation its complex conjugate, the following equation is obtained:

$$
\frac{\tilde{v}_{0}^{*}}{U_{0}-c_{0}^{*}} \frac{d}{d y}\left\{\frac{\left(U_{0}-c_{0}\right) \tilde{v}_{0 y}-U_{0 y} \tilde{v}_{0}}{\chi}\right\}=\frac{\tilde{v}_{0}}{U_{0}-c_{0}} \frac{d}{d y}\left\{\frac{\left(U_{0}-c_{0}^{*}\right) \tilde{v}_{0 y}^{*}-U_{0 y} \tilde{v}_{0}^{*}}{\chi^{*}}\right\},
$$

where

$$
\chi=T_{0}-M_{\infty}^{2}\left(U_{0}-c_{0}\right)^{2},
$$

and a superscript asterisk here denotes a complex conjugate. After some algebra, this may be written

$$
\tilde{v}_{0}^{*} \frac{d}{d y}\left[\frac{\tilde{v}_{0 y}}{\chi}\right]-\tilde{v}_{0} \frac{d}{d y}\left[\frac{\tilde{v}_{0 y}^{*}}{\chi^{*}}\right]=\tilde{v}_{0} \tilde{v}_{0}^{*}\left\{\frac{1}{U_{0}-c_{0}} \frac{d}{d y}\left[\frac{U_{0 y}}{\chi}\right]-\frac{1}{U_{0}-c_{0}^{*}} \frac{d}{d y}\left[\frac{U_{0 y}}{\chi^{*}}\right]\right\} .
$$

Writing

$$
c_{0}=R l\left\{c_{0}\right\}+i \operatorname{Im}\left\{c_{0}\right\}
$$

then the neutral state corresponds to $\operatorname{Im}\left\{c_{0}\right\} \rightarrow 0$. In this limit, (5.8) may be written 


$$
\left[\frac{\tilde{v}_{0}^{*} \tilde{v}_{0 y}-\tilde{v}_{0} \tilde{v}_{0 y}^{*}}{\chi}\right]_{y_{0}}^{y_{1}}=\frac{\tilde{v}_{0 y}\left(y_{i}\right) 2 \pi i}{U_{0 y}\left(y_{i}\right)}\left(\frac{U_{0 y}}{T}\right)_{y_{i}} .
$$

Using arguments similar to those of Duck (1990), then (i) as $\operatorname{Im}\left\{c_{0}\right\} \rightarrow 0$, the left-hand-side of the equation is zero except possibly at $y_{i}$ (where $U_{0}\left(y_{i}\right)=R l\left\{c_{0}\right\}$ ), (ii) the term in the parentheses on the left-hand-side of (5.10) must be zero at both $y=0$ and $y=1$, while (iii) the right-hand-side is clearly non-zero unless

$$
\frac{d}{d y}\left[\frac{U_{0 y}}{T_{0}}\right]_{y=y_{i}}=0
$$

Thus, in order to avoid an inconsistency, we must have (5.11).

Most importantly, there does exist a difference between the present (bounded) flow configuration, and that of unbounded flows, in that in the present situation, (5.11) is necessary if

$$
0<R l\left\{c_{0}\right\}<1
$$

i.e., a critical layer must exist inside the flow, while in the case of unbounded flows condition (5.11) only holds if the wavespeed is "subsonic", i.e., $1-1 / M_{\infty}<R l\left\{c_{0}\right\}<1+1 / M_{\infty}$. This is because (5.11) is a direct consequence of the zero velocity perturbations at the domain boundaries. There is absolutely no distinction made here between supersonic and subsonic modes. Thus any neutral inviscid mode satisfying (5.12) must be associated with a generalized inflection point. The above says nothing about neutral inviscid modes outside the range of (5.12). It is also worth noting that authors who implement Dirichlet boundary conditions (in place of radiation boundary conditions) on the disturbance terms in truncated infinite domains, may well experience difficulties computing non-inflectional supersonic modes, since the arguments above suggest that a generalized inflection point is necessary for supersonic disturbances, a condition which is clearly erroneous in the unbounded case.

Utilizing (3.5) and (3.9) in (5.11) yields,

$$
\left\{\frac{T_{0 y} \tau}{\mu_{0}^{2} T_{0}^{2}}\left[\mu_{0 T} T_{0}+\mu_{0}\right]\right\}_{y=y_{i}}=0 .
$$

If the term inside the square brackets is zero, then by Sutherland's law (2.9), we must have

$$
\left[3 T_{0}+5 C\right]_{y=y_{i}}=0,
$$

which is clearly inadmissible. Consequently, the only way that a generalized inflection point will occur is if the mean temperature profile has a local extremum. If we invoke Sutherland's law and (3.9), then we either require

$$
U_{0 y}\left(y_{i}\right)=0
$$

which is clearly not possible on account of (3.5), or

$$
U_{0}\left(y_{i}\right)=\frac{(1-r) T_{r}}{2\left[T_{r}-1\right]} .
$$


Since $T_{r}>1$, and the flow is unidirectional, then this condition cannot be satisfied unless

$$
0<U_{0}\left(y_{i}\right)<1,
$$

which implies

$$
\frac{1-\frac{\gamma-1}{2} \sigma M_{\infty}^{2}}{1+\frac{\gamma-1}{2} \sigma M_{\infty}^{2}}<r<1,
$$

(implying that the lower wall must be cooled below adiabatic conditions).

\section{Inviscid Disturbance Results}

For this set of computations, the basic profile for velocity and temperature, namely $U_{0}(y)$ and $T_{0}(y)$ was determined by means of a fourth-order Runge-Kutta scheme. The eigenvalue problem posed in (5.1) - (5.4) was solved similarly, with Newton iteration being used to update the complex wavespeed $c_{0}$ so that all boundary conditions were satisfied.

In a number of computations (specifically those for which $0 \leq R l\left\{c_{0}\right\} \leq 1$ ), it was found necessary to deform the integration contour into the complex $y$ plane (in particular below the real $y$ axis), in a manner described by Lees and Lin (1946). This was undertaken by two independent ways. The first, as suggested by Mack (1965b), involves obtaining the mean flow profile $\left(U_{0}(y), T_{0}(y)\right)$ along the real $y$ axis, and then using Taylor series expansions to obtain the mean flow profile off the real $y$ axis; this detour is made close to where $U_{0}(y)=c_{0}$. The second approach (which was generally used in preference) obtains the mean flow solution itself in the complex $y$ plane, thereby eliminating the errors associated with truncation of the Taylor series. Specifically, the mean flow was obtained for $0 \leq y \leq y_{1}$ (i.e., on the real $y$ axis), $y_{1}<y \leq y_{1}-i y_{2}, y_{1}-i y_{2}<y \leq y_{3}-i y_{2}, y_{3}-i y_{2}<y \leq y_{3}$ and thereafter back along the real axis $y_{3}<y \leq 1 . y_{1}, y_{2}, y_{3}$ were all taken to be real and positive (although this is not essential) and were chosen to avoid the computation proceeding too close to the critical layer. Comparison of results using the two approaches proved a useful check of the accuracy of our results. A further check on our results was that in addition to solving (5.1), (5.2) we also solved the adjoint system (see (7.29), (7.30) below), and also (5.5).

The first results we present is for the case $M_{\infty}=2$ and adiabatic lower wall boundary conditions (here and in all cases we took $\gamma=1.4, \sigma=0.72$ together with the Sutherland constant $C=0.5$ ). Results for $R l\left\{c_{0}\right\}$ are shown in Figure 11. The results display a number of interesting features. There appear to be many modes, which, however, can be divided into two distinct families, the first corresponding to $R l\left\{c_{0}\right\}>1$ as $\alpha \rightarrow 0$. All these modes, with the exception of one (mode I) have $R l\left\{c_{0}\right\} \rightarrow \infty$ as $\alpha \rightarrow 0$; we refer to these as the upper family. These modes all suffer a monotonic decrease in $R l\left\{c_{0}\right\}$ as $\alpha$ increases, and the results suggest that some finite value is approached in the limit $\alpha \rightarrow \infty$. The second family is defined by $R l\left\{c_{0}\right\}<0$ as $\alpha \rightarrow 0$, and the $R l\left\{c_{0}\right\}$ all increase monotonically with an increase in $\alpha$, and eventually become positive. We refer to these modes as the lower family of solutions. Again, all except the first of these modes (mode II) appear to be unbounded as $\alpha \rightarrow 0$.

A further, important feature is that in all cases for which $R l\left\{c_{0}\right\}>1$ or $R l\left\{c_{0}\right\}<0$, all these modes are neutrally stable to within machine accuracy, i.e., $\operatorname{Im}\left\{c_{0}\right\}=0$. However, 
since there can be no generalized inflection point for the mean flow under consideration, then there can be no neutral modes with $0<c_{0}<1$. Indeed, it is found that in the case of the upper family of solutions, once $R l\left\{c_{0}\right\}<1$, then these modes become stable according to our inviscid calculations, while in the case of the lower family of solutions, once $R l\left\{c_{0}\right\}>0$, then these modes become unstable according to our inviscid calculations; the location at which $R l\left\{c_{0}\right\}$ either drops below 1 or rises above 0 is marked on Figure 11 by a circle. The distribution of $\operatorname{Im}\left\{c_{0}\right\}$ for mode I is shown in Figure 12 (other upper family modes also have negative values of $\operatorname{Im}\left\{c_{0}\right\}$, but many orders of magnitude smaller). The distribution of $\operatorname{Im}\left\{c_{0}\right\}$ for mode II is shown in Figure 13 (other modes of the lower family have considerably smaller values of $\operatorname{Im}\left\{c_{0}\right\}$, although these are also positive). Next we go on to consider the aforementioned trends as $\alpha \rightarrow 0$ suggested by our numerical results.

The existence and behavior of modes I and II as $\alpha \rightarrow 0$ is easy to confirm. If we set $\alpha=0$ in (5.5), and integrate once, we obtain

$$
\left(U_{0}-c_{0}\right) \tilde{v}_{0 y}-U_{0 y} \tilde{v}_{0}=K\left[T_{0}-M_{\infty}^{2}\left(U_{0}-c_{0}\right)^{2}\right],
$$

where $K$ is an arbitrary constant. This equation may be integrated once more to yield

$$
\tilde{v}_{0}=K\left(U_{0}-c_{0}\right) \int_{0}^{y} \frac{\left[T_{0}-M_{\infty}^{2}\left(U_{0}-c_{0}\right)^{2}\right]}{\left(U_{0}-c_{0}\right)^{2}} d y .
$$

If this is to satisfy the boundary condition on $y=1$ (the boundary condition on $y=0$ is already satisfied by (6.2)), we must then have

$$
\int_{0}^{1} \frac{T_{0}}{\left(U_{0}-c_{0}\right)^{2}} d y=M_{\infty}^{2} .
$$

This relationship was solved for $c_{0}$ (for given $U_{0}(y), T_{0}(y)$, and $M_{\infty}$ ) and confirmed the numerical results as $\alpha \rightarrow 0$. Indeed, if we take a model problem, i.e.,

$$
U_{0}(y)=y, \quad T_{0}(y)=1
$$

then the solutions of $(6.3)$ are

$$
c_{0}=\frac{1 \pm\left[1+4 / M_{\infty}^{2}\right]^{1 / 2}}{2} .
$$

These modes will be discussed further in Section 7.

The behavior of modes III and higher as $\alpha \rightarrow 0$ is different, but nonetheless straightforward to confirm. In these cases we have $c_{0} \rightarrow \infty$ as $\alpha \rightarrow 0$, and a balancing of terms in (5.5) in this limit demands

$$
\begin{gathered}
c_{0}=\alpha^{-1} c_{00}+O(1), \\
\tilde{v}_{0}=\tilde{v}_{00}+O(\alpha) .
\end{gathered}
$$

The equation for $\tilde{v}_{00}$ is then

$$
\tilde{v}_{0 y y}+\frac{c_{00}^{2} M_{\infty}^{2} \tilde{v}_{00}}{T_{0}}=0
$$


If, to make further progress analytically we assume $\left|c_{00}\right| \gg 1$, implying we are focusing attention on the higher nodes (alternatively we could assume the model problem, with $T_{0}=$ $1)$, then the WKB solution is

$$
v_{00}=A T_{0}^{\frac{1}{4}}\left\{e^{i c_{00} M_{\infty} \int_{0}^{y} T_{0}^{-1 / 2} d y}-e^{-i c_{00} M_{\infty} \int_{0}^{y} T_{0}^{-1 / 2} d y}\right\},
$$

where $A$ is a constant. Here the boundary condition on $y=0$ has been applied, while the other boundary condition on $y=1$ demands

$$
c_{00}=\frac{n \pi}{M_{\infty} \int_{0}^{1} T_{0}^{-1 / 2} d y},
$$

where $n$ is any (large) positive or negative integer. This expression clearly illustrates the multiplicity of modes.

The second set of results we show is for the higher Mach number case $M_{\infty}=5$, with the adiabatic boundary conditions on the lower wall retained. Figure 14 shows a number of results for $R l\left\{c_{0}\right\}$. Although these seem much the same as the corresponding distributions for $M_{\infty}=2$, there are some differences, in particular with mode $I_{A}$, as defined in Figure 14, which initially corresponds to mode I in Figure 12. However, unlike the corresponding results for $M_{\infty}=2$, it turns out that there exist other (stable) modes in the vicinity of mode $I_{A}$. Some of these modes are shown in Figure 15 (on a magnified scale) and are denoted by $I_{B}$ and $I_{C}$. These modes were difficult to generate numerically in regions of $\alpha$ where $R l\left\{c_{0}\right\}$ was very close to unity and where the decay rates were quite small; however, the existence of these modes was confirmed using the three different formulations of the inviscid stability problem described above, together with the condition (6.3). Indeed, it is quite likely that other such modes exist, although the present investigation failed to yield any. However, it turns out that these modes are all stable, as shown in Figure 16. Note that modes $I_{A}$ and $I_{C}$ have, in places, values of $c_{0}$ exceedingly close to each other, but nonetheless distinct; this compounded the difficulty associated with these modes.

Modes II, and higher, on the other hand did exhibit the same qualitative behavior as the corresponding $M_{\infty}=2$ results. In particular, mode II becomes unstable at $\alpha \simeq 1.85$, with the distribution of $\operatorname{Im}\left\{c_{0}\right\}$ shown in Figure 17. Other higher modes of this lower family are also unstable, but with substantially smaller growth rates.

The final set of results in this section relates to the case $M_{\infty}=5$, as previously, but with the (cooled-wall) boundary condition $T_{0}(0)=1$. It is worth noting that even in this case, the uniform temperature together with a linear velocity profile is still not a proper solution of the governing equations, except in the very special limit as $\gamma \rightarrow 1$. Nonetheless, the solution does have some important properties, namely that

$$
T_{0}(y)=T_{0}(1-y)
$$

together with

$$
U_{0}(y)=1-U_{0}(1-y) .
$$

As a consequence of these symmetries, the mean flow has a single G.I.P. at $y=\frac{1}{2}$, (with $U_{0}\left(\frac{1}{2}\right)=\frac{1}{2}$ ). These properties turn out to have interesting implications for the inviscid stability of the profile. 
Figure 18 shows the variations of $R l\left\{c_{0}\right\}$ for the first eight modes. As in the previous cases, there again exist two distinct families, with all except two of the modes having the property $\left|R l\left\{c_{0}\right\}\right| \rightarrow \infty$ as $\alpha \rightarrow 0$. Furthermore, all modes are neutrally stable if $R l\left\{c_{0}\right\}<0$ or $R l\left\{c_{0}\right\}>1$. Significantly, due to the aforementioned symmetries about $y=\frac{1}{2}$, the behavior of $R l\left\{c_{0}\right\}$ for the upper family of modes is merely the mirror image of the corresponding lower family member about $y=\frac{1}{2}$. These symmetries also yield the result that $\operatorname{Im}\left\{c_{0}\right\}$ is precisely the same for corresponding modes at the same wavenumber. Figure 19 shows the distribution of $\operatorname{Im}\left\{c_{0}\right\}$ for modes I and II. These modes begin as neutrally stable, then as $R l\left\{c_{0}\right\}$ drops below unity/rises above zero, both modes become stable. As $\alpha$ increases still further, both modes have $R l\left\{c_{0}\right\} \rightarrow \frac{1}{2}$ which is reached at $\alpha \approx 5.5$. $\operatorname{Im}\left\{c_{0}\right\}$ then becomes positive, implying unstable modes. This is entirely consistent with our G.I.P. arguments, which predict neutral modes having $c_{0}=\frac{1}{2}$. Thereafter, $R l\left\{c_{0}\right\}$ for mode I increases, and decreases for mode II.

Figure 20 shows the distribution of $\operatorname{Im}\left\{c_{0}\right\}$ for modes III and IV, which over the range shown are seen to be stable. It is possible that regions of instability exist for higher $\alpha$, although the computations become increasingly difficult as $\alpha \rightarrow \infty$.

In the following section, we go on to consider regions where viscous effects are likely to become important, particularly in determining the stability properties of the flow.

\section{The Viscous Correction}

The results described in the previous sections show that there is a family of solutions of (5.1) - (5.4) comprising neutrally stable modes over a wide range of $\alpha$, which have either $c_{0}>1$, or $c_{0}<0$, and hence have no critical layer. The question then arises as to the effect of viscosity on these modes - whether it plays a stabilizing or destabilizing role in this problem. We investigate this question next.

The viscous correction to the problem arises due to the thin layers that occur as a result of the violation of the no-slip condition (and also the temperature condition) on the walls $y=0$ and $y=1$. These conditions are

$$
\tilde{u}=\tilde{v}=0 \quad \text { on } \quad y=0 \text { and } y=1,
$$

$\tilde{T}=0$ on $y=1$, while on $y=0$,

$$
\frac{\partial \tilde{T}}{\partial y}=0
$$

if the wall is insulated, and

$$
\tilde{T}=0
$$

if it is heated or cooled.

Now from (4.4) and (4.6) we see that on $y=0$ we have

$$
\tilde{u}_{0}=\frac{T_{0}(0) \tilde{p}_{0}(0)}{\gamma M_{\infty}^{2} c_{0}}
$$

and

$$
\tilde{T}_{0}=\left(\frac{\gamma-1}{\gamma}\right) T_{0}(0) \tilde{p}(0)
$$


while on $y=1$ we have

$$
\tilde{u}_{0}=\frac{T_{0}(1) \tilde{p}_{0}(1)}{\gamma M_{\infty}^{2}\left(c_{0}-1\right)}
$$

and

$$
\tilde{T}_{0}=\left(\frac{\gamma-1}{\gamma}\right) T_{0}(1) \tilde{p}_{0}(1)
$$

Thus, generally these expressions fail to satisfy the appropriate wall conditions described above.

In order to remedy this, we require a (Stokes-like) layer of thickness $O\left(\operatorname{Re}^{-\frac{1}{2}}\right)$ on both walls. Considering first the layer on $y=0$, defining

$$
Y=y \operatorname{Re}^{\frac{1}{2}}=O(1)
$$

together with

$$
\begin{aligned}
\tilde{u} & =\tilde{U}(Y)+O\left(\operatorname{Re}^{-\frac{1}{2}}\right), \\
\tilde{v} & =\operatorname{Re}^{-\frac{1}{2}} \tilde{V}(Y)+O\left(\operatorname{Re}^{-1}\right), \\
\tilde{T} & =\tilde{\tilde{T}}(Y)+O\left(\operatorname{Re}^{-\frac{1}{2}}\right), \\
\tilde{p} & =\tilde{P}(Y)+O\left(\operatorname{Re}^{-\frac{1}{2}}\right), \\
\tilde{\rho} & =\tilde{\tilde{\rho}}(Y)+O\left(\operatorname{Re}^{-\frac{1}{2}}\right),
\end{aligned}
$$

then taking $O(1)$ terms in $(4.3)-(4.7)$, we have

$$
\begin{gathered}
\frac{-i \alpha c_{0} \tilde{U}}{T_{0}(0)}=\mu_{L} \tilde{U}_{Y Y}-\frac{i \alpha \tilde{P}}{\gamma M_{\infty}^{2}}, \\
\tilde{P}_{Y}=0, \\
-i c_{0} \tilde{\tilde{\rho}}+\frac{i \tilde{U}}{T_{0}(0)}+\frac{1}{T(0)} \tilde{V}_{Y}=0, \\
\frac{-i \alpha c_{0} \tilde{\tilde{T}}}{T_{0}(0)}-\frac{\mu_{L}}{\sigma} \tilde{\tilde{T}}_{Y Y}+\left(\frac{\gamma-1}{\gamma}\right) i \alpha c_{0} \tilde{P}_{0}=0, \\
\tilde{P}=T_{0}(0) \tilde{\tilde{\rho}}+\frac{\tilde{\tilde{T}}}{T_{0}(0)},
\end{gathered}
$$

where $\mu_{L}=\mu_{0}(0)$. On account of (7.11) the pressure must be constant across this layer, and so we write

$$
\tilde{P}=\tilde{p}_{0}(0)=\tilde{p}_{L} .
$$

The solution to $(7.10)$ is then

$$
\tilde{U}=\frac{\tilde{p}_{L} T_{0}(0)}{\gamma M_{\infty}^{2} c_{0}}\left\{1-e^{-\left(\frac{-i \alpha c_{0}}{T_{0}(0) \mu_{L}}\right)^{\frac{1}{2}} Y}\right\} .
$$


In the case of an insulated wall on $y=0$, the temperature perturbation is given by (7.5) across the layer (i.e., it is constant), while for the heated or cooled wall conditions, using (7.13) we obtain

$$
\tilde{\tilde{T}}=\left(\frac{\gamma-1}{\gamma}\right) \tilde{p}_{L} T(0)\left\{1-e^{-\left(\frac{-i \alpha c_{0} \sigma}{\mu_{L} T_{0}(0)}\right)^{1 / 2} Y}\right\} .
$$

Using the continuity equation, (7.12), we obtain

$$
\begin{aligned}
\tilde{V}= & i c_{0} \tilde{p}_{L} Y-i c_{0} \tilde{p}_{L}\left(\frac{\gamma-1}{\gamma}\right)\left\{Y+\frac{b}{\left[\frac{-i \alpha c_{0} \sigma}{\mu_{L} T_{0}(0)}\right]^{\frac{1}{2}}}\left[e^{-\left(\frac{-i \alpha c_{0} \sigma}{\mu_{L} T_{0}(0)}\right)^{\frac{1}{2}} Y}-1\right]\right\} \\
& -\frac{i \tilde{p}_{L} T_{0}(0)}{\gamma M_{\infty}^{2} c_{0}}\left\{Y+\frac{1}{\left[\frac{-i \alpha c_{0}}{T_{0}(0) \mu_{L}}\right]^{\frac{1}{2}}}\left[e^{-\left(\frac{-i \alpha c_{0}}{T_{0}(0) \mu_{L}}\right)^{\frac{1}{2}} Y}-1\right]\right\},
\end{aligned}
$$

where $b=0$ in the case of an insulated wall, while $b=1$ for a cooled/heated wall. The corresponding layer on the upper wall is quite similar. Defining the lengthscale

$$
\hat{Y}=(y-1) \operatorname{Re}^{\frac{1}{2}},
$$

together with (7.9), then the results for $\hat{Y}=O(1)$ may be simply inferred from those for $Y=O(1)$ by (i) replacing ' $Y$ ' by ' $-\hat{Y}$ ', (ii) replacing ' $\tilde{p}_{L}$ ' by ' $\tilde{p}_{U}$ ' $=\tilde{p}(1)$, (iii) replacing ' $c_{0}$ ' by " $c_{0}-1$ " and (iv) replacing $\mu_{L}$ and $T_{0}(0)$ by unity. Finally the (key) result for $\tilde{V}$ is then given by

$$
\begin{aligned}
\tilde{V}= & i\left(c_{0}-1\right) \tilde{p}_{U} \hat{Y} \\
& -i\left(c_{0}-1\right) \tilde{p}_{U}\left(\frac{\gamma-1}{\gamma}\right)\left\{\hat{Y}-\frac{1}{\left[-i \alpha\left(c_{0}-1\right) \sigma\right]^{\frac{1}{2}}}\left[e^{\left[-i \alpha\left(c_{0}-1\right) \sigma\right]^{\frac{1}{2}} \hat{Y}}-1\right]\right\} \\
& -\frac{i \tilde{p}_{U}}{\gamma M_{\infty}^{2}\left(c_{0}-1\right)}\left\{\hat{Y}-\frac{1}{\left[-i \alpha\left(c_{0}-1\right)\right]^{\frac{1}{2}}}\left[e^{\left[-i \alpha\left(c_{0}-1\right)\right]^{\frac{1}{Y}} \hat{Y}}-1\right]\right\} .
\end{aligned}
$$

We shall later require the behavior of (7.18) and (7.19) as $Y \rightarrow \infty$ and $\hat{Y} \rightarrow-\infty$, respectively. We have

$$
\begin{aligned}
\tilde{V} \rightarrow & Y\left\{i c_{0} \tilde{p}_{L}-i c_{0} p_{L}\left(\frac{\gamma-1}{\gamma}\right)\right. \\
& \left.-i \frac{\tilde{p}_{L}}{\gamma M_{\infty}^{2} c_{0}}\right\} \\
& +\frac{i c_{0} \tilde{p}_{L} b\left(\frac{\gamma-1}{\gamma}\right)}{\left[\frac{-i \alpha c_{0} \sigma}{T_{0}(0) \mu_{L}}\right]^{\frac{1}{2}}}+\frac{i \tilde{p}_{L} T_{0}(0)}{\gamma M_{\infty}^{2} c_{0}\left[\frac{-i \alpha c_{0}}{T_{0}(0) \mu_{L}}\right]^{\frac{1}{2}}}
\end{aligned}
$$




$$
\rightarrow \tilde{v}_{0 L} Y+\tilde{v}_{1 L} \quad \text { as } Y \rightarrow \infty
$$

and

$$
\begin{gathered}
\tilde{V} \rightarrow \hat{Y}\left\{i\left(c_{0}-1\right) \tilde{p}_{U}-\frac{i\left(c_{0}-1\right) \tilde{p}_{U}\left(\frac{\gamma-1}{\gamma}\right)}{\left[-i \alpha\left(c_{0}-1\right) \sigma\right]^{\frac{1}{2}}}-\frac{i \tilde{p}_{U}}{\gamma M_{\infty}^{2}\left(c_{0}-1\right)\left[-i \alpha\left(c_{0}-1\right)\right]^{\frac{1}{2}}}\right\} \\
-\frac{i\left(c_{0}-1\right) \tilde{p}_{U}\left(\frac{\gamma-1}{\gamma}\right)}{\left[-i \alpha\left(c_{0}-1\right) \sigma\right]^{1 / 2}}-\frac{i \tilde{p}_{U}}{\gamma M_{\infty}^{2}\left(c_{0}-1\right)\left[-i \alpha\left(c_{0}-1\right)\right]^{1 / 2}} \\
\rightarrow \tilde{v}_{0 U} \hat{Y}+\tilde{v}_{1 U} \quad \text { as } \hat{Y} \rightarrow-\infty .
\end{gathered}
$$

We now seek the leading-order viscous correction term to (5.1), (5.2) for $y=O(1)$, using the expansions (4.14). The variables $\tilde{v}_{1}$ and $\tilde{p}_{1}$ turn out to be determined by an inhomogeneous form of (5.1) and (5.2), namely

$$
\begin{gathered}
\tilde{v}_{1 y}-\frac{U_{0 y} \tilde{v}_{1}}{U_{0}-c_{0}}-\frac{i \tilde{p}_{1}}{U_{0}-c_{0}}\left[\frac{T_{0}-M_{\infty}^{2}\left(U_{0}-c_{0}\right)^{2}}{\gamma M_{\infty}^{2}}\right]=c_{1} R_{1}, \\
\tilde{p}_{1 y}+\frac{i \alpha^{2} \gamma M_{\infty}^{2}}{T_{0}}\left(U_{0}-c_{0}\right) \tilde{v}_{1}=c_{1} R_{2},
\end{gathered}
$$

where

$$
\begin{gathered}
R_{1}=\frac{U_{0 y} \tilde{v}_{0}}{\left(U_{0}-c_{0}\right)^{2}}+\frac{i \tilde{p}_{0} T_{0}}{\gamma M_{\infty}^{2}\left(U_{0}-c_{0}\right)^{2}}+\frac{i \tilde{p}_{0}}{\gamma}, \\
R_{2}=\frac{i \gamma M_{\infty}^{2} \alpha^{2} \tilde{v}_{0}}{T_{0}} .
\end{gathered}
$$

The boundary conditions on this system are also inhomogeneous, namely

$$
\begin{aligned}
& \tilde{v}_{1}(0)=\tilde{v}_{1 L} \\
& \tilde{v}_{1}(1)=\tilde{v}_{1 U},
\end{aligned}
$$

(the boundary conditions for $\tilde{p}_{1 y}$ may be obtained from (7.25), although this is not necessary for the following), where $\tilde{v}_{1 L}$ and $\tilde{v}_{1 U}$ are defined by (17.21) and (7.23) respectively.

To obtain $c_{1}$, we use the condition of solvability of the system (7.24) - (7.28). For this we require the adjoint to the system; if we denote $v^{+}$and $p^{+}$as the adjoint functions, then these are to be determined by

$$
\begin{gathered}
v_{y}^{+}+\frac{U_{0 y} v^{+}}{U_{0}-c_{0}}+\frac{i \alpha^{2}\left(U_{0}-c_{0}\right) \gamma M_{\infty}^{2}}{T_{0}} p^{+}=0, \\
p_{y}^{+}-\frac{i v^{+}}{U_{0}-c_{0}}\left[\frac{T_{0}-M_{\infty}^{2}\left(U_{0}-c_{0}\right)^{2}}{\gamma M_{\infty}^{2}}\right]=0 .
\end{gathered}
$$

The boundary conditions to be applied to this system are that

$$
p^{+}(0)=p^{+}(1)=0 .
$$

$c_{1}$ is then given by

$$
c_{1}=\frac{v^{+}(1) \tilde{v}_{1 U}-v^{+}(0) \tilde{v}_{1 L}}{\int_{0}^{1}\left[R_{1} \bar{v}^{+}+R_{2} \bar{p}^{+}\right] d y}
$$


where $\bar{v}^{+}$and $\bar{p}^{+}$are the complex conjugates to $v^{+}$and $p^{+}$respectively.

Given the nature of the solution for $v^{+}, p^{+}, \tilde{v}_{1 U}, \tilde{v}_{1 L}, R$, and $R_{2}$ when $c_{0}$ is real, it is easy to show that we must have

$$
c_{1}=\operatorname{Im}\left\{c_{1}\right\}( \pm 1+i)
$$

where the positive sign is taken for $c_{0}>0$, the negative sign is taken for $c_{0}<0$; note that $\operatorname{Im}\left\{c_{1}\right\}>0$ for instability. However, in all the computations we performed, without exception, we found $\operatorname{Im}\left\{c_{1}\right\}<0$. Results for $\operatorname{Im}\left\{c_{1}\right\}$ for the adiabatic case with $M_{\infty}=2$ (modes I and II) are shown in Figure 21. The distribution becomes unbounded as $\alpha \rightarrow 0$, and also as $\alpha \rightarrow \alpha_{0}$, the point at which $c_{0} \rightarrow 1$ or 0 ; these distributions are typical of all modes. Figure 22 shows the corresponding distributions for the adiabatic case with $M_{\infty}=5$, and exhibit, qualitatively, the same behavior. (The corresponding computation for $M_{\infty}=5, T_{0}(0)=1$ was also carried out, and yielded qualitatively the same picture.) We shall defer discussion of the limit $\alpha \rightarrow \alpha_{0}$ until the following section, but let us now consider the limit as $\alpha \rightarrow 0$, of modes I and II from which it appears that our expansions cease to be uniformly valid. This is most clearly seen by the apparently singular behavior of the viscous correction to the complex wavespeed as $\alpha \rightarrow 0$, together with the $O\left(\alpha^{-\frac{1}{2}}\right)$ growth of the wall-layer thickness in this limit (see (7.10) for example). Thus when $\alpha=O\left(\mathrm{Re}^{-1}\right)$ the wall layers will fill the entire channel, and the disturbances become purely viscous in nature.

Specifically, if we write

$$
\alpha=\operatorname{Re}^{-1} \bar{\alpha},
$$

then the governing equations (4.3) - (4.6) reduce to

$$
\begin{gathered}
-i c \tilde{\rho}+i \rho_{0} \tilde{u}+\rho_{0} \tilde{v}+i U_{0} \tilde{\rho}+\tilde{v} \rho_{0 y}=0, \\
\bar{\alpha} \rho_{0}\left[-i c \tilde{u}+i U_{0} \tilde{u}+\tilde{v} U_{0 y}\right]+\frac{i \bar{\alpha} \tilde{p}}{\gamma M_{c o}^{2}} \\
=\mu_{0} \tilde{u}_{y y}+\mu_{0 T} \tilde{T} U_{0 y}+\mu_{0 T} T_{0 y} \tilde{u}_{y}, \\
\tilde{p}_{y}=0, \\
\bar{\alpha} \rho_{0}\left[-i c \tilde{T}+i U_{0} \tilde{T}+\tilde{v} T_{0 y}\right]-\left(\frac{\gamma-1}{\gamma}\right)\left[i \bar{\alpha} U_{0}-i \bar{\alpha} c\right] \tilde{p} \\
=\frac{\mu_{0}}{\sigma} \tilde{T}_{y y}+\frac{\mu_{0 T}}{\sigma} T_{0 y} \tilde{T}_{y}+\frac{\tilde{T} \mu_{0 T}}{\sigma} T_{0 y y}+\frac{\tilde{T}_{y} \mu_{0 T}}{\sigma} \\
+\tilde{T} \mu_{0 T T} T_{0 y}+\tilde{T} \mu_{0 T}(\gamma-1) M_{\infty}^{2} U_{0 y}^{2},
\end{gathered}
$$

where it has been implicitly assumed that viscosity is a function of temperature only. The problem then reduces to a basically viscous system.

However, for modes III and higher, $c_{0}=O\left(\alpha^{-1}\right)$ as $\alpha \rightarrow 0$, and since the Stokes layer thickness remains $O\left(R^{-\frac{1}{2}}\right)$, it does not fill the entire channel. Additionally, as $\alpha \rightarrow 0$, $\operatorname{Im}\left\{c_{1}\right\}=O\left(\alpha^{-1}\right)$, and so the expansions (4.14) remain valid.

Returning to Figure 9, we note the general excellent agreement between the values of $\sqrt{R e} \operatorname{Im}\{c\}$ obtained numerically, and $\operatorname{Im}\left\{c_{1}\right\}$ obtained from the above asymptotic theory, 
for the case $M_{\infty}=2$ (mode II). The values of $c_{r}$ obtained using the two approaches are almost indistinguishable. We do note a deviation between the results as $c_{0} \rightarrow 0$, caused by the (expected) breakdown in the asymptotic theory in this limit (and as $c_{0} \rightarrow 1$ ). This aspect is investigated in the following section.

\section{The Nature of the Solution as $c_{0} \rightarrow 0\left(\right.$ or $\left.c_{0} \rightarrow 1\right)$}

We consider here the nature of the complex wavespeed as $c_{0} \rightarrow 0$ (the results for $c_{0} \rightarrow 1$ may be simply inferred from those of $c_{0} \rightarrow 0$ ). Our previous results indicate that as $c_{0} \rightarrow 0$, (with $\alpha \rightarrow \alpha_{0}$ ) then (i) $c_{1}$, the viscous correction to the wavespeed becomes unbounded, (ii) the leading order wavespeed $c_{0}$ becomes non-neutral as $\alpha$ increases above $\alpha_{0}$, and (iii) the thickness of the wall layer increases. Thus this region is likely to be a regime of some interest, to which we now focus our attention.

More specifically, guided by our numerical results, if we write

$$
\alpha=\alpha_{0}+\varepsilon \alpha_{1},
$$

where

$$
\varepsilon=\mathrm{Re}^{-\frac{1}{3}}
$$

then we expect

$$
c=\varepsilon \hat{c}_{1}+O\left(\varepsilon^{\frac{3}{2}}\right) .
$$

The solution in the "core," i.e., away from the wall layers is then expected to develop in the form

$$
\begin{aligned}
& \tilde{v}=\hat{v}_{0}(y)+\varepsilon \hat{v}_{1}(y)+\cdots, \\
& \tilde{p}=\hat{p}_{0}(y)+\varepsilon \hat{p}_{1}(y)+\cdots .
\end{aligned}
$$

The leading order system is then

$$
\hat{v}_{0 y}-\frac{U_{0 y} \hat{v}_{0}}{U_{0}}-\frac{i \hat{p}_{0}}{\gamma M_{\infty}^{2} U_{0}}\left[T_{0}-M_{\infty}^{2} U_{0}^{2}\right]=0
$$

or symbolically

$$
\mathcal{L}_{1}\left\{\hat{v}_{0}, \hat{p}_{0}\right\}=0
$$

and

$$
\frac{\hat{p}_{0 y}}{\gamma M_{\infty}^{2}}+i \alpha_{0}^{2} \frac{U_{0} \hat{v}_{0}}{T_{0}}=0
$$

or symbolically

$$
\mathcal{L}_{2}\left\{\hat{v}_{0}, \hat{p}_{0}\right\}=0
$$

together with

$$
\hat{v}_{0}(0)=\hat{v}_{0}(1)=0 .
$$

Notice that (8.6) and (8.8) imply that $\hat{p}_{0}=O\left(y^{3}\right)$ as $y \rightarrow 0$; this system effectively determines the value(s) of $\alpha_{0}$ for which $c_{0}=0$. Turning to the next order system, we find

$$
\mathcal{L}_{1}\left\{\hat{v}_{1}, \hat{p}_{1}\right\}=U_{0 y} \frac{\hat{v}_{0} \hat{c}_{1}}{U_{0}^{2}}+\frac{i \hat{p}_{0}}{\gamma M_{\infty}^{2} U_{0}^{2}} \hat{c}_{1}\left[T_{0}+M_{\infty}^{2} U_{0}^{2}\right]=\hat{c}_{1} \hat{R}_{1},
$$




$$
\mathcal{L}_{2}\left\{\hat{v}_{1}, \hat{p}_{1}\right\}=\frac{i \alpha_{0}^{2} \hat{c}_{1} \hat{v}_{0}}{T_{0}}-\frac{2 i \alpha_{0} \alpha_{1} U_{0} \hat{v}_{0}}{T_{0}}=\hat{c}_{1} \hat{R}_{2}+\alpha_{1} \hat{R}_{3} .
$$

The boundary conditions to be applied to this system are

$$
\hat{v}_{1}(1)=0, \quad \hat{v}_{1}(0)=\Lambda \hat{p}_{1}(0),
$$

where $\Lambda$ is to be determined later. Using conditions of solvability, we must have that

$$
\hat{c}_{1}=\frac{-\hat{v}^{+}(0) \Lambda \hat{p}_{1}(0)-\alpha_{1} \int_{0}^{1} \hat{R}_{3} \hat{p}^{+} d y}{\int_{0}^{1}\left[\hat{R}_{1} \hat{v}^{+}+\hat{R}_{2} \hat{p}^{+}\right] d y},
$$

where $\hat{p}^{+}(y), \hat{v}^{+}(y)$ are the adjoint functions to (8.6), (8.8), namely those determined by (7.29), (7.30) with $\alpha=\alpha_{0}, c_{0}=0$.

We now consider the effect of the wall layers. The upper wall layer remains of thickness $O\left(\operatorname{Re}^{-\frac{1}{2}}\right)$ and as such plays no role to this order. The interesting changes are related to the lower wall layer, where now we must have

$$
\tilde{Y}=\varepsilon^{-1} y=O(1)
$$

as the crucial scale, wherein to leading order

$$
(\tilde{u}, \tilde{v}, \tilde{p})=\left(\tilde{U}_{0}, \varepsilon \tilde{V}_{0}, \varepsilon \tilde{P}_{0}\right) .
$$

Taking the leading order terms in the continuity and momentum equations we find

$$
\begin{gathered}
i \tilde{U}_{0}+\tilde{V}_{0 \tilde{Y}}=0, \\
\tilde{P}_{0 \tilde{Y}}=0, \\
i \alpha_{0}\left[U_{0}^{\prime}(0) \tilde{Y}-\hat{c}_{1}\right] \tilde{U}_{0}+\alpha_{0} U_{0}^{\prime}(0) \tilde{V}_{0}=\hat{U}_{0 \tilde{Y} \tilde{Y}}-i \alpha_{0} \tilde{P}_{0},
\end{gathered}
$$

with boundary conditions

$$
\tilde{U}_{0}(0)=\tilde{V}_{0}(0)=0
$$

and as $\tilde{Y} \rightarrow \infty$.

$$
\tilde{U}_{0} \rightarrow-i \hat{v}_{0 y}(0),
$$

where the latter condition arises from a proper matching with the $y=O(1)$ solution. If we now differentiate (8.19) with respect to $\tilde{Y}$, invoke (8.17) and (8.18) we find

$$
\tilde{U}_{0 \tilde{Y} \tilde{Y} \tilde{Y}}-i \alpha_{0}\left[U_{0}^{\prime}(0) \tilde{Y}-\hat{c}_{1}\right] \tilde{U}_{0 \tilde{Y}}=0,
$$

whose solution is

$$
\tilde{U}_{0 \check{Y}}=C_{1} A i\left\{\left[i \alpha_{0} U_{0}^{\prime}(0)\right]^{\frac{1}{3}}\left(\tilde{Y}-\bar{c}_{1}\right)\right\},
$$

where $C_{1}$ is a constant to be determined, and $\bar{c}_{1}=\hat{c}_{1} / U_{0}^{\prime}(0)$.

Utilizing the boundary condition on $\tilde{Y}=0$, and also evaluating (8.19) on $\tilde{Y}=0$ leads to

$$
\tilde{U}_{0}=\frac{i \alpha_{0} \tilde{P}_{0} \int_{0}^{\tilde{Y}} A i\left\{\left[i \alpha_{0} U_{0}^{\prime}(0)\right]^{\frac{1}{3}}\left(\tilde{Y}-\bar{c}_{1}\right)\right\} d \tilde{Y}}{\left[i \alpha_{0} U_{0}^{\prime}(0)\right]^{\frac{1}{3}} A i^{\prime}\left\{-\left[i \alpha_{0} U_{0}^{\prime}(0)\right]^{\frac{1}{3}} \bar{c}_{1}\right\}} .
$$


$\tilde{V}_{0}$ may now be determined by continuity (8.12) together with $(8.20)$, and is given by

$$
\tilde{V}_{0}=\frac{\tilde{P}_{0} \int_{0}^{\tilde{Y}} \int_{0}^{\tilde{Y}} A i\left\{\left[i \alpha_{0} U_{0}^{\prime}(0)\right]^{\frac{1}{3}}\left(\tilde{Y}-\bar{c}_{1}\right)\right\} d \tilde{Y} d \tilde{Y}}{\left[i \alpha_{0} U_{0}^{\prime}(0)\right]^{\frac{1}{3}} A i^{\prime}\left\{-\left[i \alpha_{0} U_{0}^{\prime}(0)\right]^{\frac{1}{3}} \bar{c}_{1}\right\}}
$$

Correct matching with the core then requires

$$
\tilde{P}_{0}=\frac{-\left[i \alpha_{0} U_{0}^{\prime}(0)\right]^{\frac{1}{3}} \hat{v}_{0 y}(0) A i^{\prime}\left\{-\left[i \alpha_{0} U_{0}^{\prime}(0)\right]^{\frac{1}{3}} \bar{c}_{1}\right\}}{\alpha_{0}^{2} \int_{0}^{\infty} A i\left\{\left[i \alpha_{0} U_{0}^{\prime}(0)\right]^{\frac{1}{3}}\left(\hat{Y}-\bar{c}_{1}\right)\right\} d \tilde{Y}}
$$

together with

$$
\tilde{P}_{0}=\hat{p}_{1}(0)
$$

and

$$
\left[\tilde{V}_{0}(\tilde{Y})-\tilde{Y} \tilde{V}_{0}(\hat{Y})\right]_{\tilde{Y} \rightarrow \infty}=\hat{v}_{1}(0)
$$

It is these conditions that then enable us to determine $\Lambda\left(\hat{c}_{1}\right)$ introduced previously,

$$
\Lambda=\frac{\alpha_{0}^{2} \int_{0}^{\infty} \int_{\infty}^{\bar{Y}} A i\left\{\left[i \alpha_{0} U_{0}^{\prime}(0)\right]^{\frac{1}{3}}\left(\hat{Y}-\bar{c}_{1}\right) d \tilde{Y} d \tilde{Y}\right.}{\left[i \alpha U_{0}^{\prime}(0)\right]^{\frac{1}{3}} A i^{\prime}\left\{-\left[i \alpha_{0} U_{0}^{\prime}(0)\right]^{\frac{1}{3}} \bar{c}_{1}\right\}}
$$

The procedure is then to determine a solution to (8.11), (8.12) consistent with (8.13), (8.14), and (8.27). However, the problem for $\hat{c}_{1}$ is highly nonlinear, and as such is a difficult numerical task. However, if we suppose $\left|\alpha_{1}\right| \rightarrow \infty$, then

$$
\hat{c}_{1}=\frac{-\alpha_{1} \int_{0}^{1} \hat{R}_{3} \hat{p}^{+} d y}{\int_{0}^{1}\left[\hat{R}_{1} \hat{v}^{+}+\hat{R}_{2} \hat{p}^{+}\right] d y}+O\left(\alpha^{-\frac{1}{2}}\right),
$$

which, on account of the nature of $\hat{p}^{+}, \hat{v}^{+}, \hat{R}_{1}, \hat{R}_{2}, \hat{R}_{3}$ can be shown to be purely real to leading order; indeed it is interesting to note that although this sytem does admit complex values of $\hat{c}_{1}$ for $\alpha_{1}=O(1)$, it appears that the above fails to capture the non-neutral nature of the inviscid modes for $\alpha>\alpha_{0}$.

However, the reason for this is clearly illustrated by considering the nature of the inviscid system (5.1) - (5.2) in the limit as $\alpha \rightarrow \alpha_{0}$. Suppose that we set

$$
\alpha=\alpha_{0}+\tilde{\alpha},
$$

where

$$
|\tilde{\alpha}| \ll\left|\alpha_{0}\right|,
$$

then we expect expansions of the form

$$
\begin{gathered}
\tilde{v}_{0}=\hat{v}_{0}+\tilde{\alpha} \hat{v}_{1}+\tilde{\alpha}^{2} \hat{v}_{2}+O\left(\tilde{\alpha}^{3}\right), \\
\tilde{p}_{0}=\hat{p}_{0}+\tilde{\alpha} \hat{p}_{1}+\tilde{\alpha}^{2} \hat{p}_{2}+O\left(\tilde{\alpha}^{3}\right), \\
c_{0}=\tilde{\alpha} \hat{c}_{1}+\tilde{\alpha}^{2} \hat{c}_{2}++O\left(\tilde{\alpha}^{3}\right) .
\end{gathered}
$$


The leading order system is

$$
\begin{aligned}
& \mathcal{L}_{1}\left\{\hat{v}_{0}, \hat{p}_{0}\right\}=0, \\
& \mathcal{L}_{2}\left\{\hat{v}_{0}, \hat{p}_{0}\right\}=0,
\end{aligned}
$$

(see $(8.7),(8.9))$, with

$$
\hat{v}_{0}(0)=\hat{v}_{0}(1)=0
$$

At the next order, $\hat{v}_{1}$ and $\hat{p}_{1}$ are again determined by means of (8.12), although we shall defer discussion of the boundary conditions to be applied to this system, and turn instead to consideration of the $O\left(\tilde{\alpha}^{2}\right)$ terms. We find

$$
\begin{aligned}
\mathcal{L}_{1}\left\{\hat{v}_{2}, \hat{p}_{2}\right\}= & \frac{U_{0 y} \hat{v}_{0} \hat{c}_{1}^{2}}{U_{0}^{3}}+\frac{\hat{v}_{0} U_{0 y} \hat{c}_{2}}{U_{0}^{2}}+\frac{U_{0 y} \hat{v}_{1} \hat{c}_{1}}{U_{0}^{2}}+\frac{i \hat{p}_{1} \hat{c}_{1}}{U_{0}^{2}}\left[T_{0}-M_{\infty}^{2} U_{0}^{2}\right] \\
& +\frac{i \hat{p}_{0}}{U_{0}^{2}}\left\{\left[\hat{c}_{2}+\frac{\hat{c}_{1}}{U_{0}}\right]\left[T_{0}-M_{\infty}^{2} U_{0}^{2}\right]+2 M_{\infty}^{2} U_{0} \hat{c}_{1}^{2}\right\}+2 M_{\infty}^{2} \hat{c}_{1} \hat{p}_{1} \\
= & R_{a}, \\
\mathcal{L}_{2}\left\{\hat{v}_{2}, \hat{p}_{2}\right\}= & -\frac{i}{T_{0}} U_{0} \hat{v}_{0}-\frac{2 i \alpha_{0}}{T_{0}} U_{0} \hat{v}_{1}+\frac{2 i \alpha_{0} \hat{c}_{1}}{T_{0}} \hat{v}_{0}-\frac{i \alpha_{0}^{2}}{T_{0}}\left[-\hat{c}_{1} \hat{v}_{1}-\hat{c}_{2} \hat{v}_{0}\right] \\
= & R_{b} .
\end{aligned}
$$

However, the expansions above are not uniformly valid as $y \rightarrow 0$, since $U_{0}(y)=O(y)$ in this limit, and hence a breakdown to our approximations must occur, specifically when $y=O(\tilde{\alpha})$. If we set

$$
y=\tilde{\alpha} \bar{Y}, \quad \bar{Y}=O(1),
$$

then $\hat{v}_{0}$ develops in the following manner (that may be demonstrated to be correct a posteriori)

$$
\hat{v}_{0}=\tilde{\alpha} \Phi_{0}(\bar{Y})+\tilde{\alpha}^{2} \ln \tilde{\alpha} \Phi_{1}(\bar{Y})+\tilde{\alpha}^{2} \Phi_{2}(\bar{Y})+\cdots
$$

It is simple to show that

$$
\Phi_{0}(\bar{Y})=-\frac{K_{0}}{c_{1}} \bar{Y}
$$

where $K_{0}$ is some constant; this is consistent with (8.33). We shall defer discussion of $\Phi_{1}(\bar{Y})$ until later, while $\Phi_{2}(\bar{Y})$ is given by

$$
\begin{aligned}
\frac{\Phi_{2 \bar{Y}}}{\left[U_{0}^{\prime}(0) \bar{Y}-\hat{c}_{1}\right]}-\frac{U_{0}^{\prime}(0) \Phi_{2}}{\left[U_{0}^{\prime}(0) \bar{Y}-\hat{c}_{1}\right]^{2}}= & \frac{K_{1} T_{0}(0)}{\left[U_{0}^{\prime}(0) \bar{Y}-\hat{c}_{1}\right]^{2}}+\frac{K_{0} \bar{Y} T_{0}^{\prime}(0)}{T_{0}(0)\left[U_{0}^{\prime}(0) \bar{Y}-\hat{c}_{1}\right]^{2}} \\
& -\frac{\hat{c}_{2} K_{0} T_{0}(0)}{\hat{c}_{1}\left[U_{0}^{\prime}(0) \bar{Y}-\hat{c}_{1}\right]^{2}}-\frac{\frac{1}{2} U_{0}^{\prime \prime}(0) \bar{Y}^{2} K_{0}}{\hat{c}_{1}\left[U_{0}^{\prime}(0) \bar{Y}-\hat{c}_{1}\right]^{2}},
\end{aligned}
$$

where $K_{1}$ is a constant. 
Asymptotic analysis of (8.39) reveals that as $\bar{Y} \rightarrow \infty$

$$
\Phi_{2} \sim \lambda_{0} \bar{Y} \ln \bar{Y}+\lambda_{1} \bar{Y}+\lambda_{2} \ln \bar{Y}+\lambda_{3}+\cdots,
$$

where the $\lambda_{i}$ are all constants. It is easy to show (see below) that

$$
\lambda_{0}=\frac{K_{0} T_{0}^{\prime}(0)}{U_{0}^{\prime}(0) T_{0}(0)}-\frac{U_{0}^{\prime \prime}(0) K_{0} \hat{c}_{1}}{\left[U_{0}^{\prime}(0)\right]^{2}} .
$$

However, if $\Phi_{2}$ is to make a proper match with the $Y=O(1)$ region, we must have

$$
\Phi_{1}=\lambda_{0}\left[U_{0}^{\prime}(0)\right] \bar{Y}
$$

It turns out that the logarithms are crucial for the generation of non-neutral values of $c_{0}$. The solution of (8.39) may be written

$$
\begin{aligned}
\Phi_{2} & =\lambda_{0} U_{0}^{\prime}(0)\left[U_{0}^{\prime}(0) \bar{Y}-\hat{c}_{1}\right] \int_{0}^{\bar{Y}} \frac{d \bar{Y}}{\left[U_{0}^{\prime}(0) \bar{Y}-\hat{c}_{1}\right]}+\cdots \\
& =\lambda_{0}\left[U_{0}^{\prime}(0) \bar{Y}-\hat{c}_{1}\right] \ln \left[\frac{\hat{c}_{1}-U_{0}^{\prime}(0) \bar{Y}}{\hat{c}_{1}}\right]+\cdots,
\end{aligned}
$$

where we have only retained terms which are of immediate concern. The crucial significance of (8.44) is the presence of the $+i \pi$ jump in the value of the logarithm (Mack, 1984, for example), as the point $\bar{Y}=\hat{c}_{1} / U_{0}^{\prime}(0)$ is traversed; it is this that generates an imaginary component to $\Phi_{2}$, given as $Y \rightarrow \infty$ by

$$
\Phi_{2}^{i} \sim \lambda_{0} \pi\left[U_{0}^{\prime}(0) \bar{Y}-c_{0}\right]
$$

(here and below a superscript ' $i$ ' denotes an imaginary component).

Returning to our discussion of the core region, we see we must have that

$$
\hat{v}_{1}(0)=\hat{v}_{1}(1)=0,
$$

and consequently $\hat{c}_{1}$ is determined by the expression (8.14), with $\Lambda=0$. On account of the nature of $\hat{R}_{1}, \hat{R}_{2}, \hat{R}_{3}, \hat{v}^{+}, \hat{p}^{+}$we expect $\hat{c}_{1}$ to be a real quantity.

We are now in a position to specify the following boundary conditions

$$
\hat{v}_{2}(1)=0, \quad \hat{v}_{2}^{i}(0)=-\lambda_{0} \hat{c}_{1} \pi,
$$

and thus $\hat{c}_{2}^{i}$ may be determined, in principle, by means of the condition

$$
\int_{0}^{1}\left[R_{a}^{r} \hat{v}^{+}+R_{b}^{i} p^{+}\right] d y=\hat{v}(0) \lambda_{0} \hat{c}_{1} \pi
$$

where superscript ' $r$ ' denotes a real part.

Unfortunately, we see from (8.41), that $\lambda_{0}$ is dependent upon $T_{0}^{\prime}(0)$ and $U_{0}^{\prime \prime}(0)$, and since both these quantities are zero in the case of an adiabatic lower wall, then in this case we must consider the expressions (8.31) up to $O\left(\tilde{\alpha}^{3}\right)$ in order to determine a value for the 
imaginary component of the wavespeed as $\tilde{\alpha} \rightarrow 0$; however, since the general technique is well established above, we do not carry this out in this paper.

The key result, therefore, is then that $\operatorname{Im}\left\{c_{0}\right\}$ is $O\left(\tilde{\alpha}^{2}\right)$ in general (but $O\left(\tilde{\alpha}^{3}\right)$ in the insulated wall case), and as a consequence we do not expect to obtain non-neutral values of $\hat{c}_{1}$, from (8.14) as $\alpha_{1} \rightarrow \infty$ (this would only be expected in a study of the $O\left(\varepsilon^{2}\right)$ terms of the $c$ expansion, in general, and the $O\left(\varepsilon^{3}\right)$ terms in the case of an insulated lower wall). The above also clearly illustrates how $c_{0}$ is only complex for values of $\alpha>\alpha_{0}$, since for $\alpha<\alpha_{0}$ the 'jump' in the value of the logarithm is not present.

\section{A Change of Boundary Conditions}

It is curious that the (confined) flow profile under consideration in this paper has such different stability characteristics from those of the (external) boundary layer. This leads us to question the nature of these fundamental differences.

One obvious candidate for investigation is the effect of the boundedness of the domain (i.e., the impermeability condition imposed on two walls). In an attempt to assess this effect, we considered the problem where the impermeability constraint on the upper wall $(y=1)$, is replaced by one of radiation, while retaining the same basic profile. Specifically, we replace (5.3) by

$$
\tilde{v}_{0}(0)=0
$$

(as before), but with

$$
\frac{\partial \tilde{v}_{0}}{\partial y}+\nu \tilde{v}_{0}=0 \quad \text { on } y=1
$$

where

$$
\nu= \pm \alpha\left[1-M_{\infty}^{2}\left(1-c_{0}\right)^{2}\right]^{\frac{1}{2}} .
$$

Here the sign of $\nu$ is chosen to ensure that $R l\{\nu\}>0$. Indeed this amounts to considering the piecewise continuous flow which consists of our basic Couette flow for $0 \leq y<1$, and uniform flow $\left(U_{0}(y)=T_{0}(y)=1\right)$ over $1<y<\infty$. This may be shown using the arguments used by Drazin and Reid (1981) who considered incompressible piecewise linear velocity profiles and imposed continuity of pressure at $y=1$.

The system defined by $(5.1)-(5.2),(9.1)-(9.2)$ is solved numerically by the techniques used in obtaining the results of Section 6 (i.e., a Runge-Kutta shooting scheme in conjunction with a Newton iteration scheme to iterate on $c_{0}$ ).

Results for one example were obtained, namely for $M_{\infty}=2$, with adiabatic conditions on the temperature at $y=0$. Results for $R l\left\{c_{0}\right\}$ are shown in Figure 23. Two modes were found, the first (mode I) originates at $\alpha=0$, with a wavespeed $c_{0}=1-1 / M_{\infty}=0.5$, and as such is typical of so-called "first modes" observed in supersonic boundary layer stability studies (e.g., Mack, 1987). Mode II, on the other hand, originates with a wavespeed $c_{0} \approx 1.48$, and consequently is more typical of the bounded flow modes observed in previous sections of this paper. Figure 24 shows the variation of $\operatorname{Im}\left\{c_{0}\right\}$ with $\alpha$. Mode I is unstable over the entire range of $\alpha$ shown, while mode II is neutrally stable up to $\alpha \approx 3.5$, at which point $R l\left\{c_{0}\right\}$ drops below unity, and $\operatorname{Im}\left\{c_{0}\right\}$ becomes negative, indicating a stable mode. We were unable to find other modes over the range of $\alpha$ considered, particularly modes with 
$R l\left\{c_{0}\right\}<0$. Thus it appears that the imposition of radiation type boundary conditions results in a hybrid situation, with stability characteristics similar to those found in both bounded and unbounded flows.

\section{Conclusions}

In this paper, we considered the linear stability of compressible plane Couette flow. Our numerical results of the full governing equations are clearly in agreement with the predictions of Morawetz $(1952,1954)$, and also there is a qualitative agreement with the work of Glatzel (1989), although the latter author considered a model basic flow.

The main thrust of this paper has been directed towards modes which have been predominantly inviscid in nature. It appears that these are the most important, since they may become unstable, while our studies indicate that viscosity plays a generally stabilizing role, throughout.

The expansions of the assumed form (4.11) are typical of solutions which are predominantly inviscid, but have viscous corrections (see also Morawetz 1952). In Section 8 we studied the important regimes where $c_{0} \rightarrow 0$ (or $c_{0} \rightarrow 1$ ) in which the first two terms in the series become comparable, a critical layer forms close to one of the walls, and the viscous layer thickens to $O\left(\mathrm{Re}^{-\frac{1}{3}}\right)$. These are the "exceptional" cases referred to by Morawetz (1952).

In Section 9, it was shown how, to a large extent, it is the boundary conditions imposed on disturbances, that determines the nature of the stability of the flow. Indeed, earlier in the paper, in Section 4 it was shown in the study of the G.I.P. condition that it is important to impose correct (i.e., radiation type) boundary conditions in the case of unbounded flows to avoid the (erroneous) requirement that a G.I.P. is necessary for the existence of supersonic neutral modes. 


\section{References}

Ashpis, D. E. and Erlebacher, G. 1989 in Instability and Transition II, Ed. M.Y. Hussaini \& R.G. Voigt, Springer Verlag, 145.

Case, K. M. 1960, Phys. Fluids 3, 143.

Case, K. M. 1961, J. Fluid Mech. 10, 420.

Davey, A. 1973, J. Fluid Mech. 57, 369.

Deardorff, J. W. 1963, J. Fluid Mech. 15, 623.

Dikii, L. A. 1964, Prikl. Mat. Mekh. 28, 389. Translated in J. Appl. Math. Mech. 28, 479 (1964).

Duck, P. W. 1990, J. Fluid Mech. 214, 611.

Eckaus, W. 1965, Studies in nonlinear stability theory. Springer Tracts in Natural Philosophy, Vol. 6, Berlin: Springer-Verlag.

Ellingsen, T., Gjevik, B, and Palm, L. 1970, J. Fluid Mech. 40, 97.

Gallagher, A. P. 1974, J. Fluid Mech. 65, 24.

Gallagher, A. P. and Mercer, A. 1962, J. Fluid Mech. 13, 91.

Gallagher, A. P. and Mercer, A. 1964, J. Fluid Mech. 18, 350.

Girard, J. J. 1988, Ph.D. Dissertation, Washington State University.

Glatzel 1989, J. Fluid Mech 202, 515-541.

Greenough, J., Riley, J. J., Soetrismo, M. and Eberhardt, S. 1988, AIAA paper 89-0372.

Grohne, D. 1954, Z. Angew. Math. Mech. 34, 344. Translated in Tech. Memor. Nat. Adv. Comm. Aero. Wash., No. 1417 (1957).

Hains, F. D. 1967, Phys. Fluids 10, 2079.

Herbert, T. 1990 in "Instability and Transition", edited by M.Y. Hussaini and R.G. Voigt, Springer-Verlag, New York.

Jackson, T. and Grosch, C. E. 1989, J. Fluid Mech., 208, 609.

Kuwahara, S. 1967, Phys. Fluids Suppl. II 10, 5115.

Lees, L. and Lin, C. C. 1946, NACA Tech. Note 1115.

Lessen, M. and Cheifetz, M.G. 1975., Phys. Fluids, 18, 939. 
Macaraeg, M.G. and Streett, C.L. 1989, Phys. Fluids A, 1, 1305.

Mack, L. M. 1963, Space Programs Summary No. 3, 7-27, p. 297, J.P.L. Pasadena, CA.

Mack, L. M. 1965a, AGARDograph 97, Part 1.329.

Mack, L. M. 1965b, in "Methods in Computational Physics," (B. Alder, S. Fernbach, and M. Rotenberg, eds.), Vol. 4, 247, Academic, NY.

Mack, L. M. 1969, J.P.L., Pasadena, CA, Document No. 90-277, Rev. A.

Mack, L. M. 1984, AGARD Report No. 704, 3-1.

Mack, L. M. 1987, Proc. Workshop on the Stability of Time Dependent and Spatially varying Flows, Springer-Verlag.

Mack, L. M. 1990, Theo. and Comp. Fluid Dyn., 2, 97.

Morawetz, C. S. 1952, J. Rat. Mech. and Anal. 1, 579.

Morawetz, C. S. 1959 J. Math. and Phys. 1.

Papageorgiou, D. 1990. Theor. and Comp. Fluid Dyn. 1, 327.

Reichardt, H. 1956, Z. Angew. Math. Mech. 36, 526.

Reynolds, W. C. and Dutten, M. C. 1967, J. Fluid Mech. 27, 465.

Robertson, J. M. 1959., Proc. 6th Mid. Western Conf. on Fluid Mech., University of Texas, 169.

Romanov V. A. 1973, Funkcional Anal. i Proložen. 7, No. 2, 62, Translated in Functional Anal. and Its Applics., 7, 137 (1973).

Shivamoggi, B.K. 1962, Acta Mechanica 44, 327.

Synge, J. L. 1938, Semi-centenn., Publ. Amer. Math. Soc. 2, 227.

Tam, C. K. W. and Hu, F. Q. 1989a, J. Fluid Mech. 201, 447.

Tam, C. K. W. and Hu, F. Q. 1989b, J. Fluid Mech. 203, 51.

Taylor, G. I. 1936, Proc. Roy. Soc. A 157, 546.

Wasow, W. 1953, J. Res. Nat. Bur. Stand. 51, 195.

Watson, J. 1960, J. Fluid Mech. 9, 371.

Zhuang, M., Kubota, T. and Dimotakis, P. E. 1990, Phys. Fluids A 2, 599. 

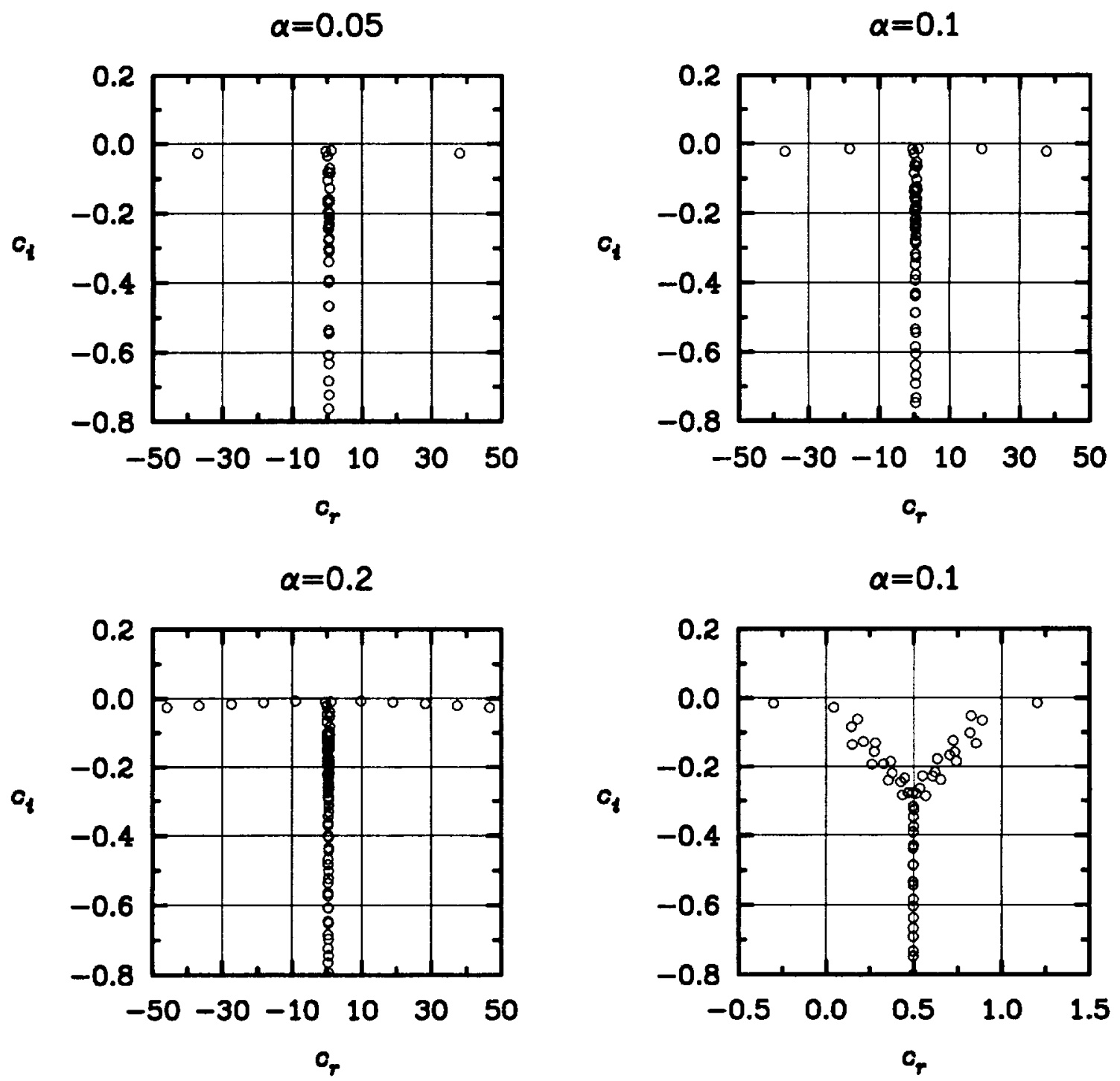

Figure 1: Spectrum at $M_{\infty}=2, \operatorname{Re}=2 \times 10^{5}$, at 100pts. (a) $\alpha=0.05$, (b) $\alpha=0.10$, (c) $\alpha=0.20$ and (d) blowup of Figure $1 \mathrm{~b}$. 

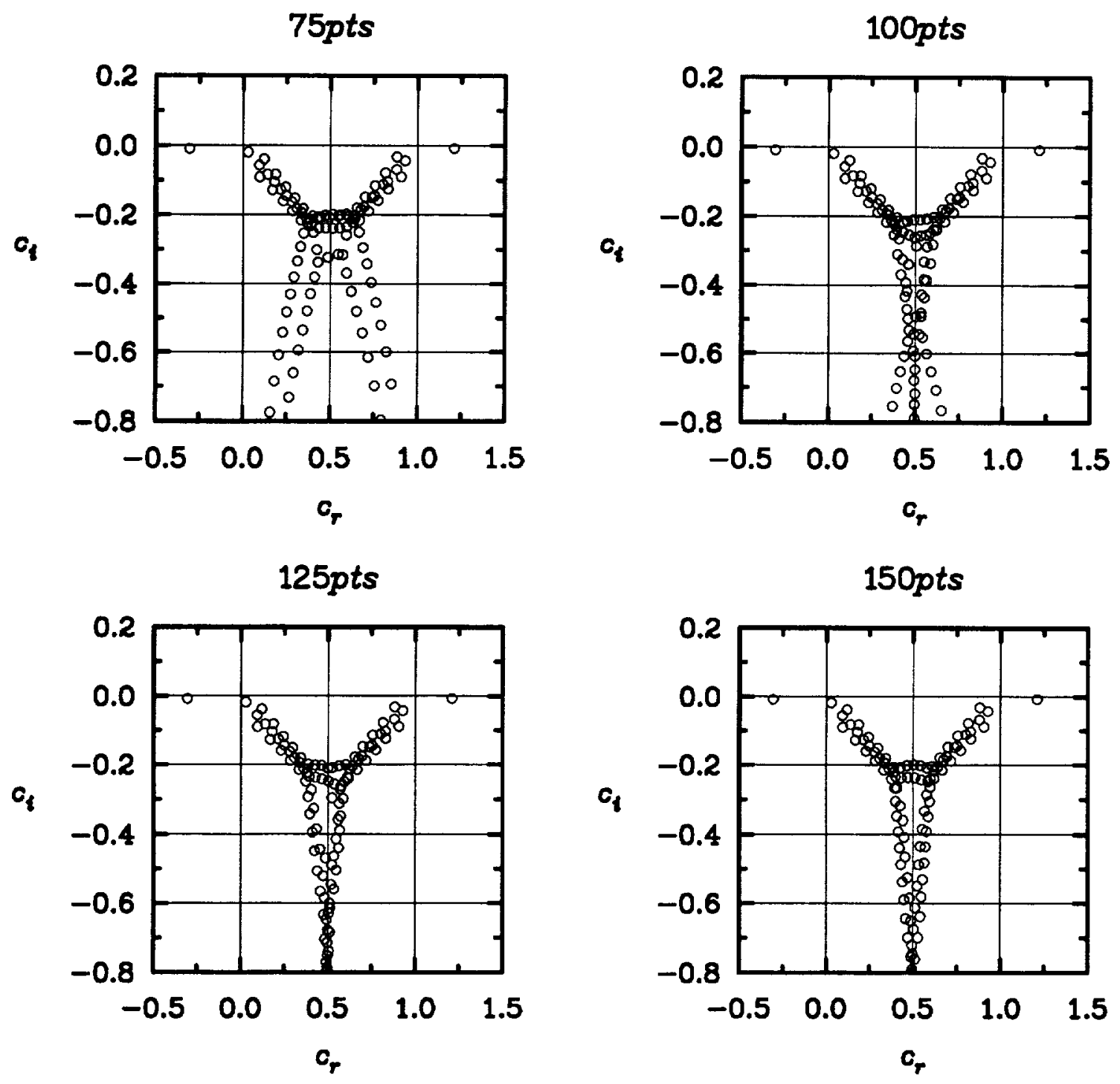

Figure 2: Phase velocity spectrum at $M_{\infty}=2, \alpha=0.1, \mathrm{Re}=7 \times 10^{5}$. (a) $75 \mathrm{pts}$, (b) 100 pts, (c) 125 pts, (d) 150 pts. 

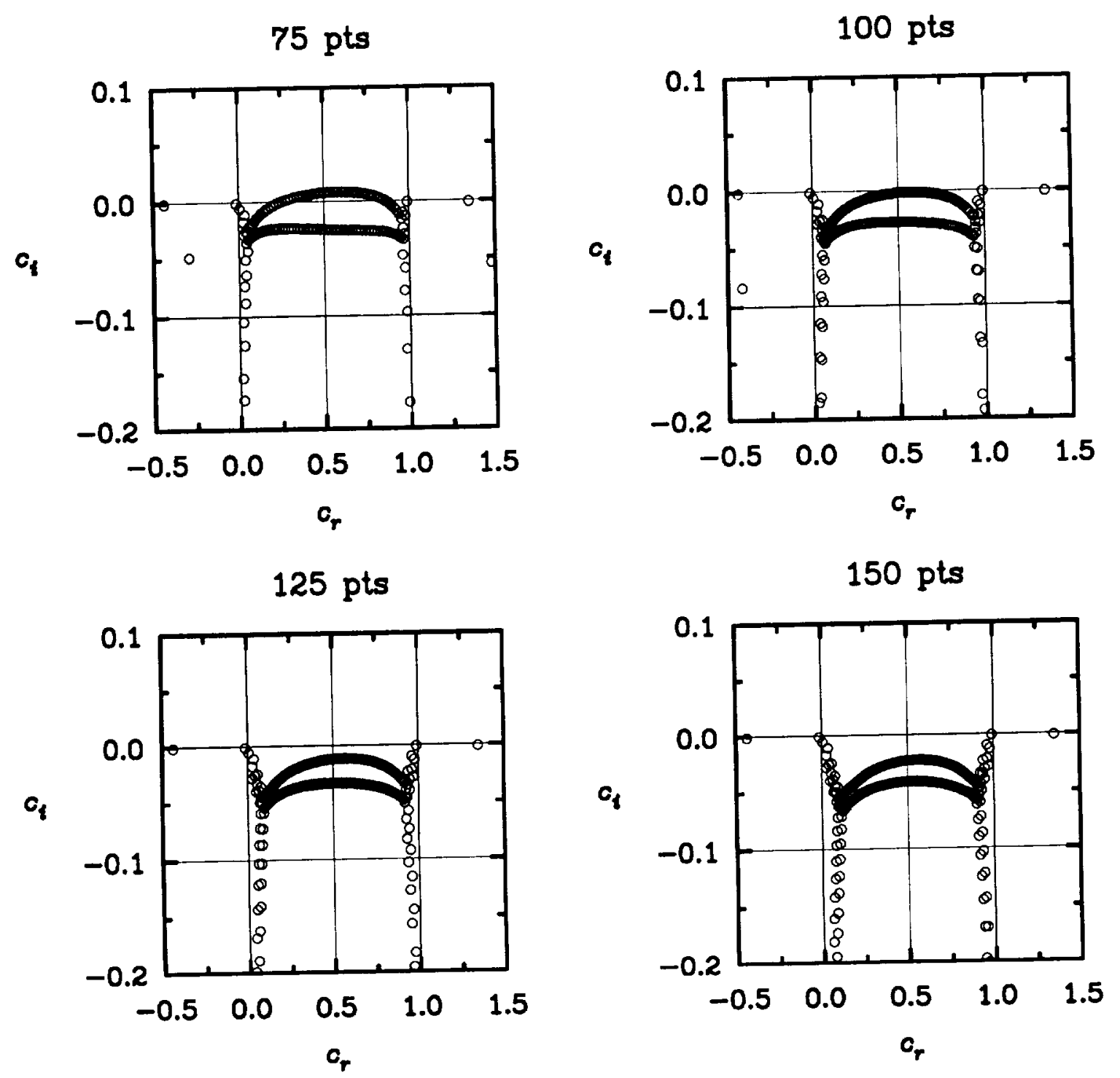

Figure 3: Phase velocity spectrum at $M_{\infty}=2, \alpha=3.5, \mathrm{Re}=7 \times 10^{5}$. (a) $75 \mathrm{pts}$, (b) 100 pts, (c) 125 pts, (d) 150 pts. 

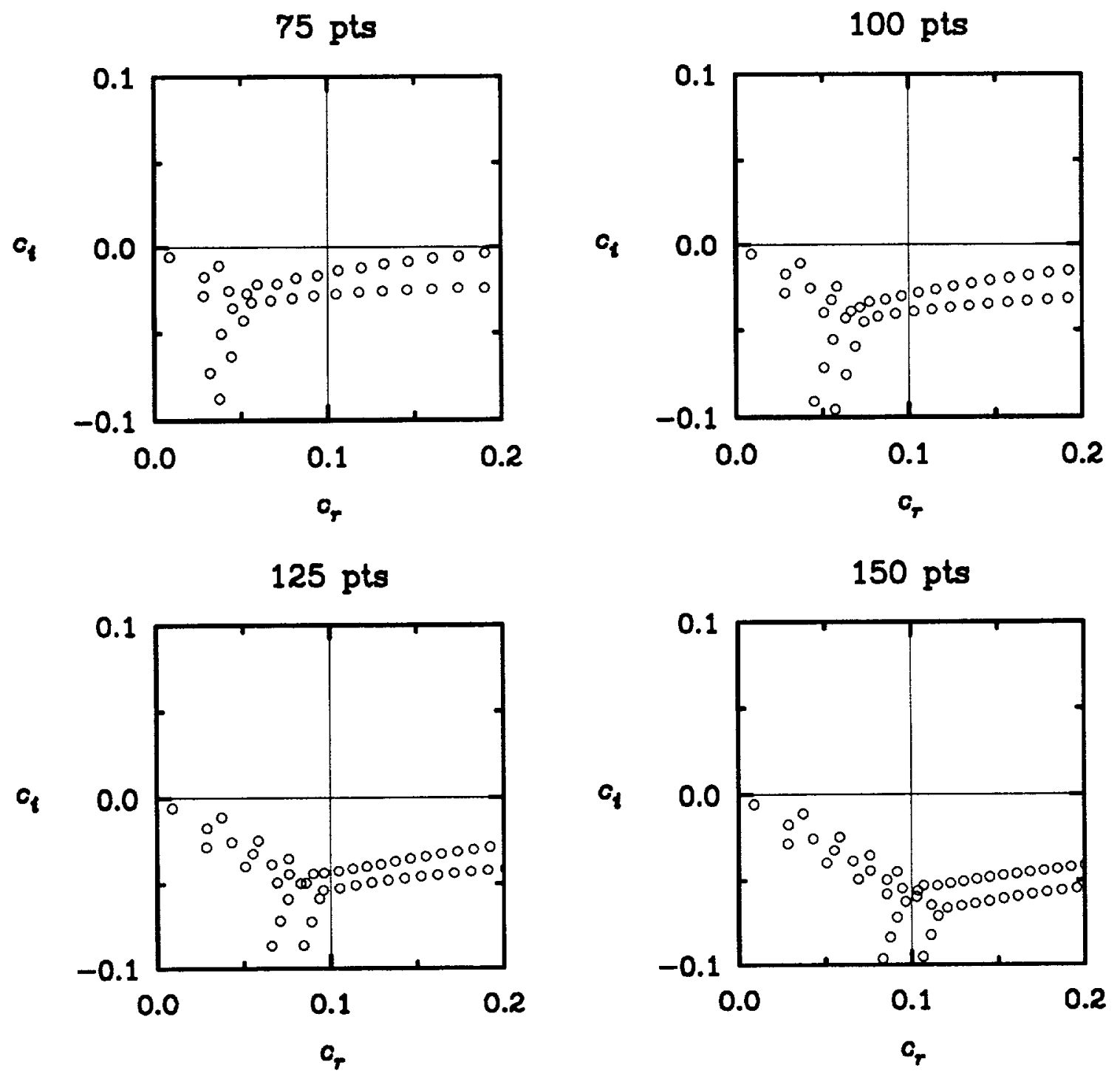

Figure 4: Blow up of Figure 3. Same parameters as Figure 3. 

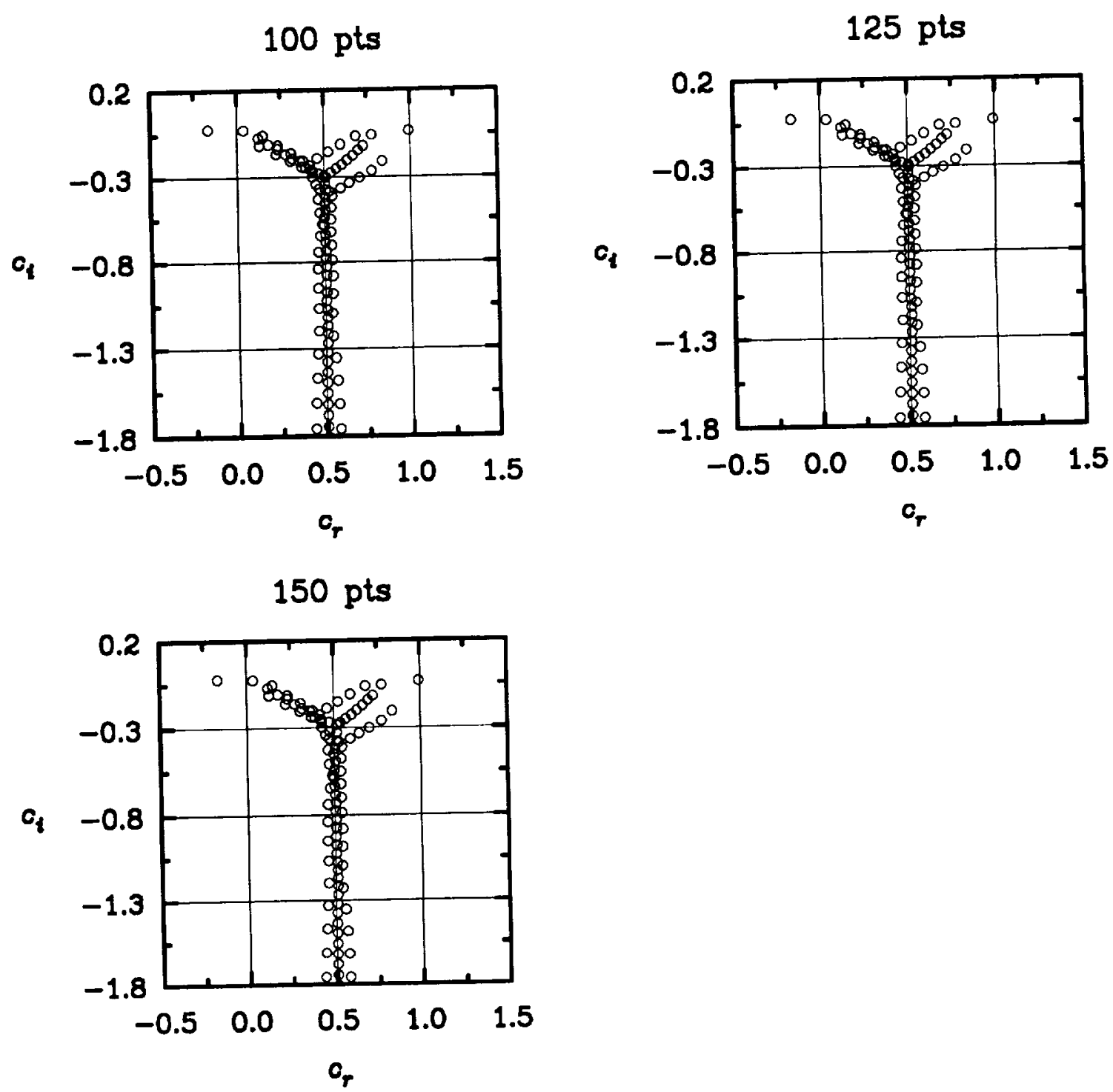

Figure 5: Phase velocity spectrum at $M_{\infty}=5, \alpha=0.1, \operatorname{Re}=2 \times 10^{6}$. (a) $100 \mathrm{pts}$, (b) 125 pts, (c) 150 pts. 



Figure 6: Phase velocity spectrum at $M_{\infty}=5, \alpha=0.1, \operatorname{Re}=5 \times 10^{6}$. (a) $100 \mathrm{pts}$, (b) 125 pts, (c) 150 pts. 

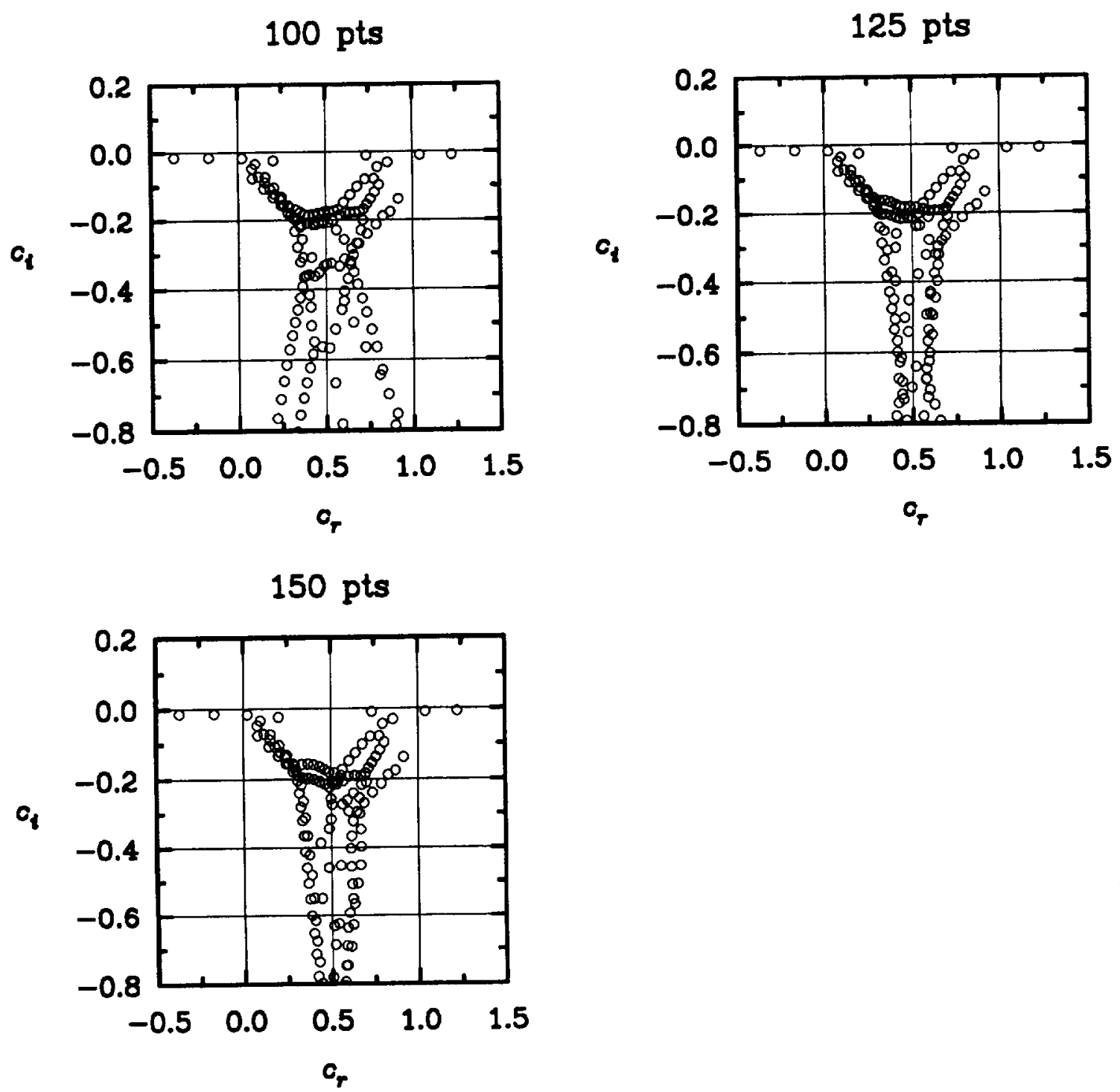

Figure 7: Phase velocity spectrum at $M_{\infty}=5, \alpha=3.5, \operatorname{Re}=2 \times 10^{5}$. (a) $100 \mathrm{pts}$, (b) 125 pts, (c) 150 pts. 



Figure 8: Phase velocity spectrum at $M_{\infty}=5, \alpha=3.5, \operatorname{Re}=7 \times 10^{5}$. (a) $75 \mathrm{pts}$, (b) 100 pts, (c) $125 \mathrm{pts,} \mathrm{(d)} 150 \mathrm{pts}$. 


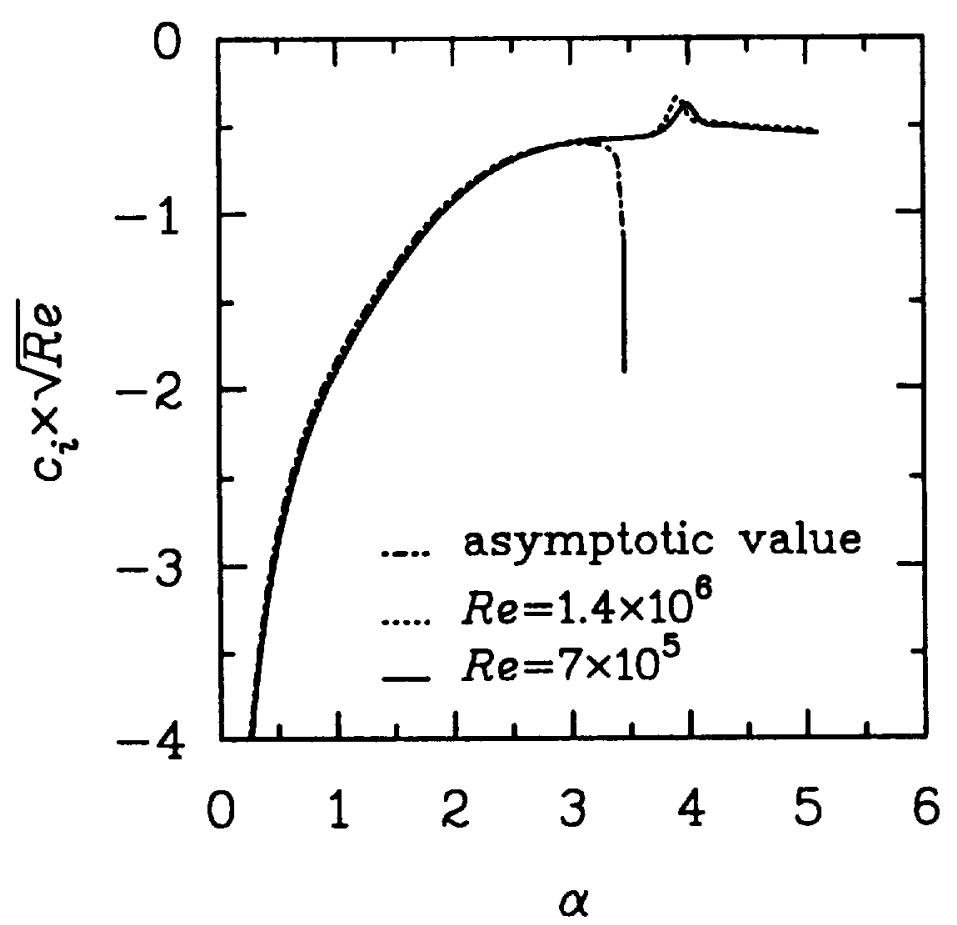

Figure 9: $c_{i} \sqrt{R e}$ as a function of $\alpha$ for $M_{\infty}=2$ (mode II), and the two Reynolds numbers $\operatorname{Re}=7 \times 10^{5}$ and $\operatorname{Re}==1.4 \times 10^{6}$. The third curve is $\operatorname{Im}\left\{c_{1}\right\}$ obtained from asymptotic theory.

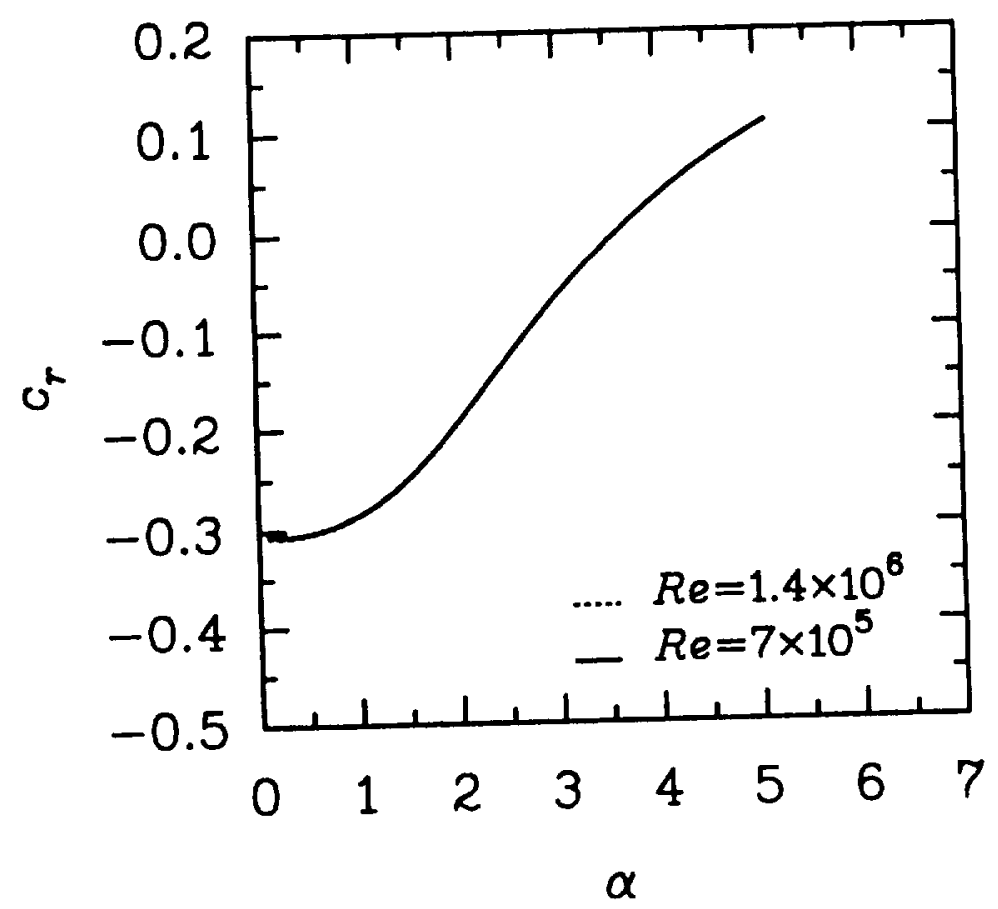

Figure 10: $c_{r}$ as a function of $\alpha$ for $M_{\infty}=2$ (mode II), and the two Reynolds numbers Re $=7 \times 10^{5}$ and $\operatorname{Re}==1.4 \times 10^{6}$. 


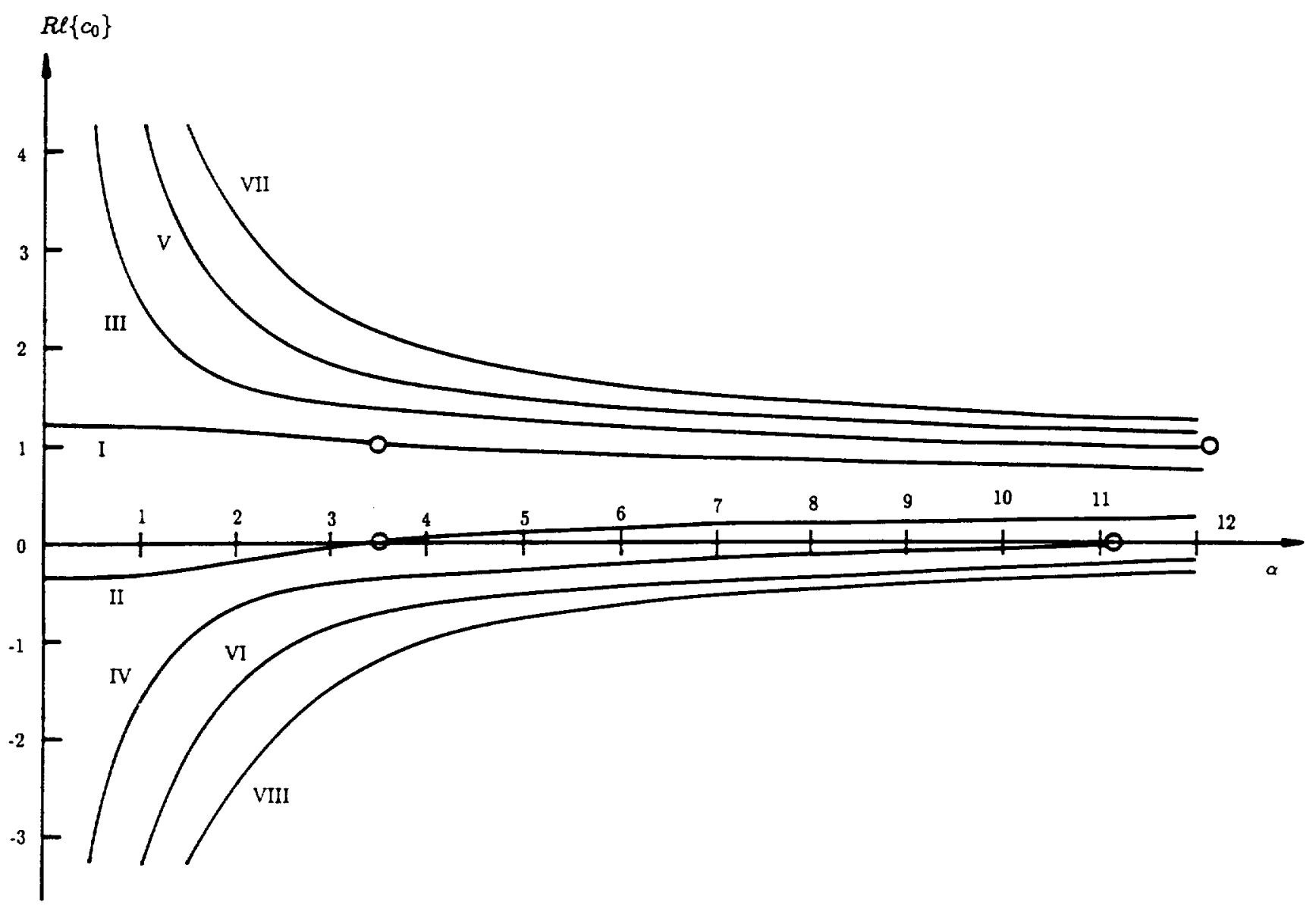

Figure 11: $R l\left\{c_{0}\right\}$ as a function of $\alpha$ for $M_{\infty}=2$, adiabatic lower wall. 


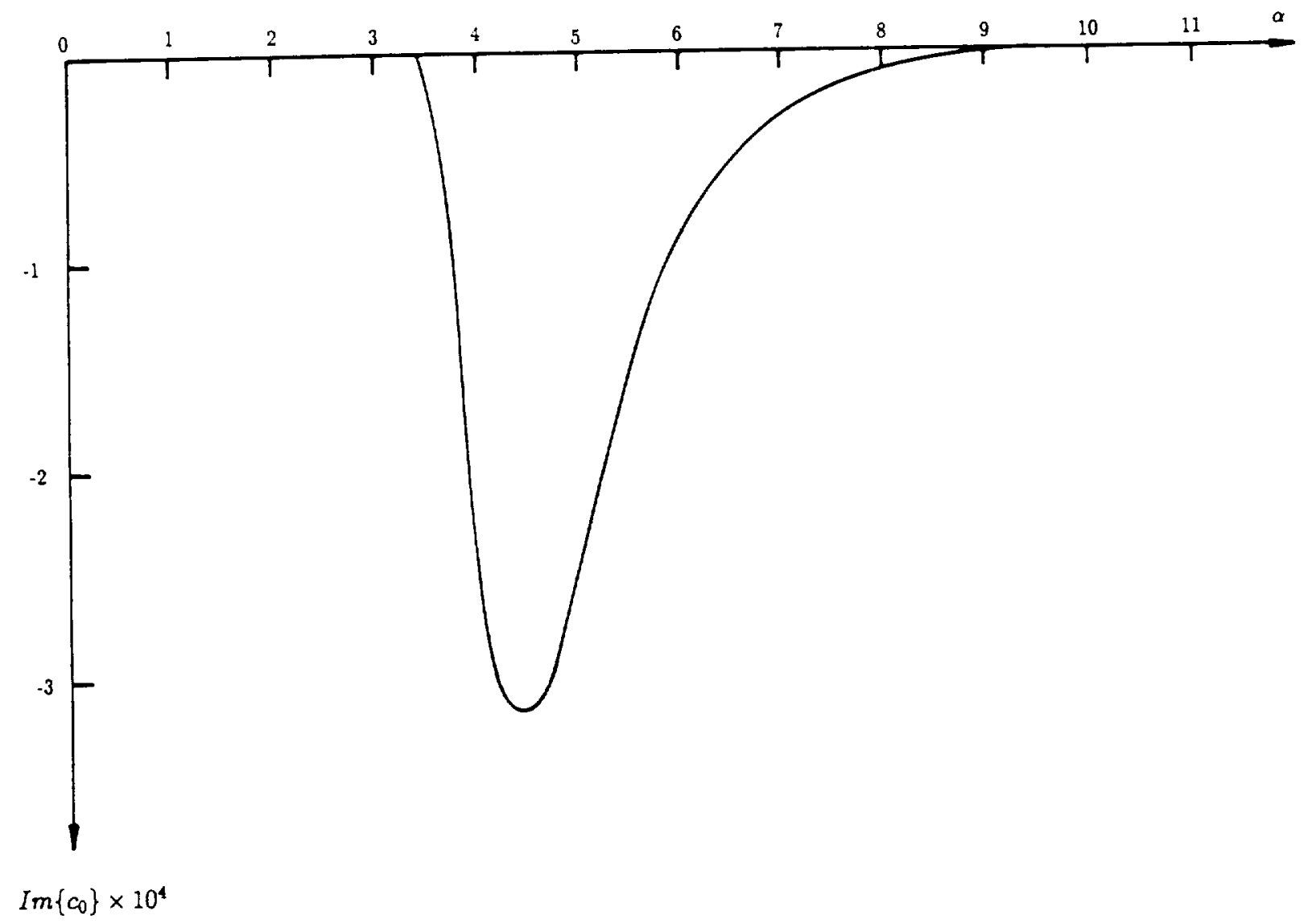

Figure 12: $\operatorname{Im}\left\{c_{0}\right\}$ as a function of $\alpha$ for $M_{\infty}=2$, adiabatic lower wall, mode I. 


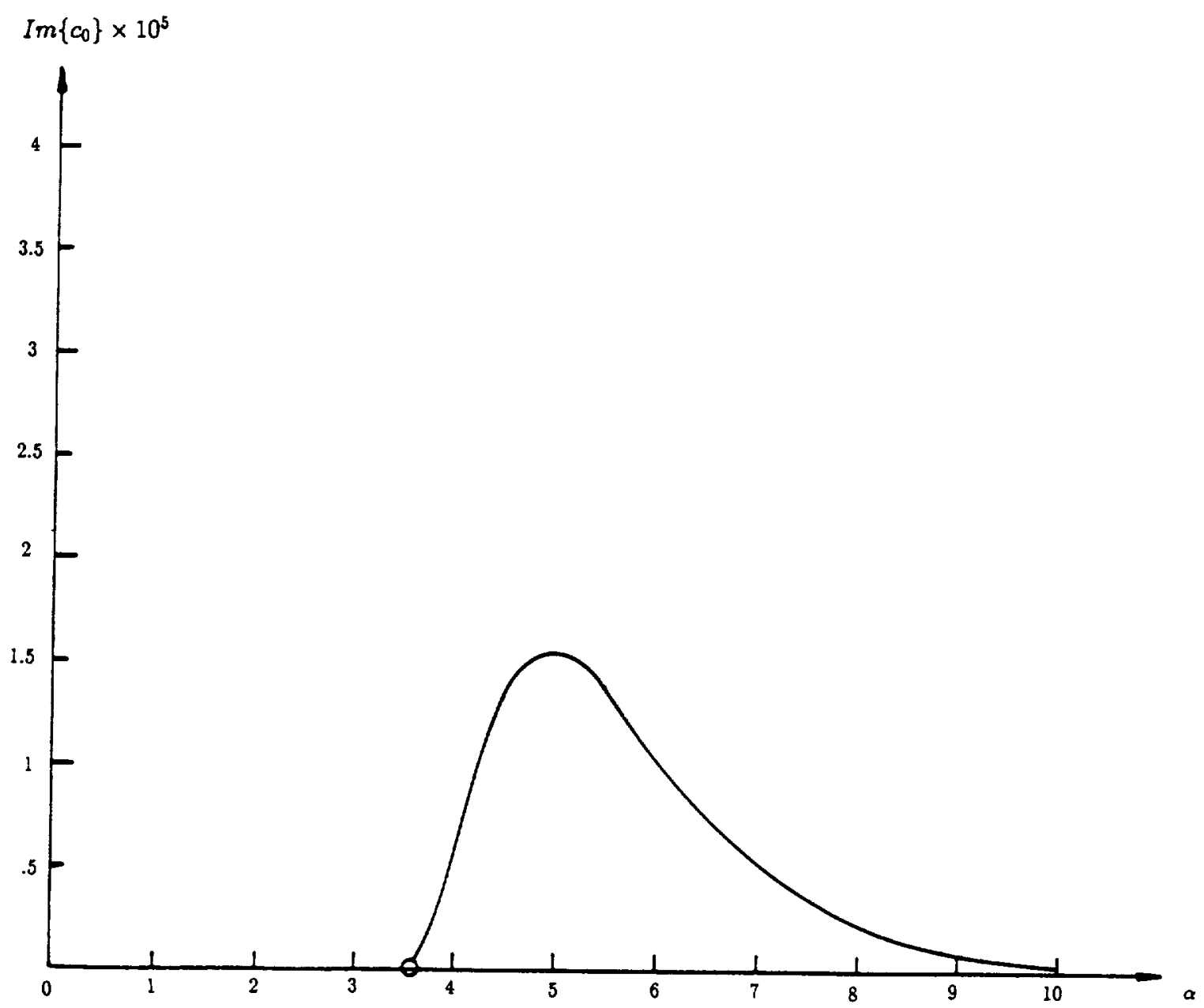

Figure 13: $\operatorname{Im}\left\{c_{0}\right\}$ as a function of $\alpha$ for $M_{\infty}=2$, adiabatic lower wall, mode II. 


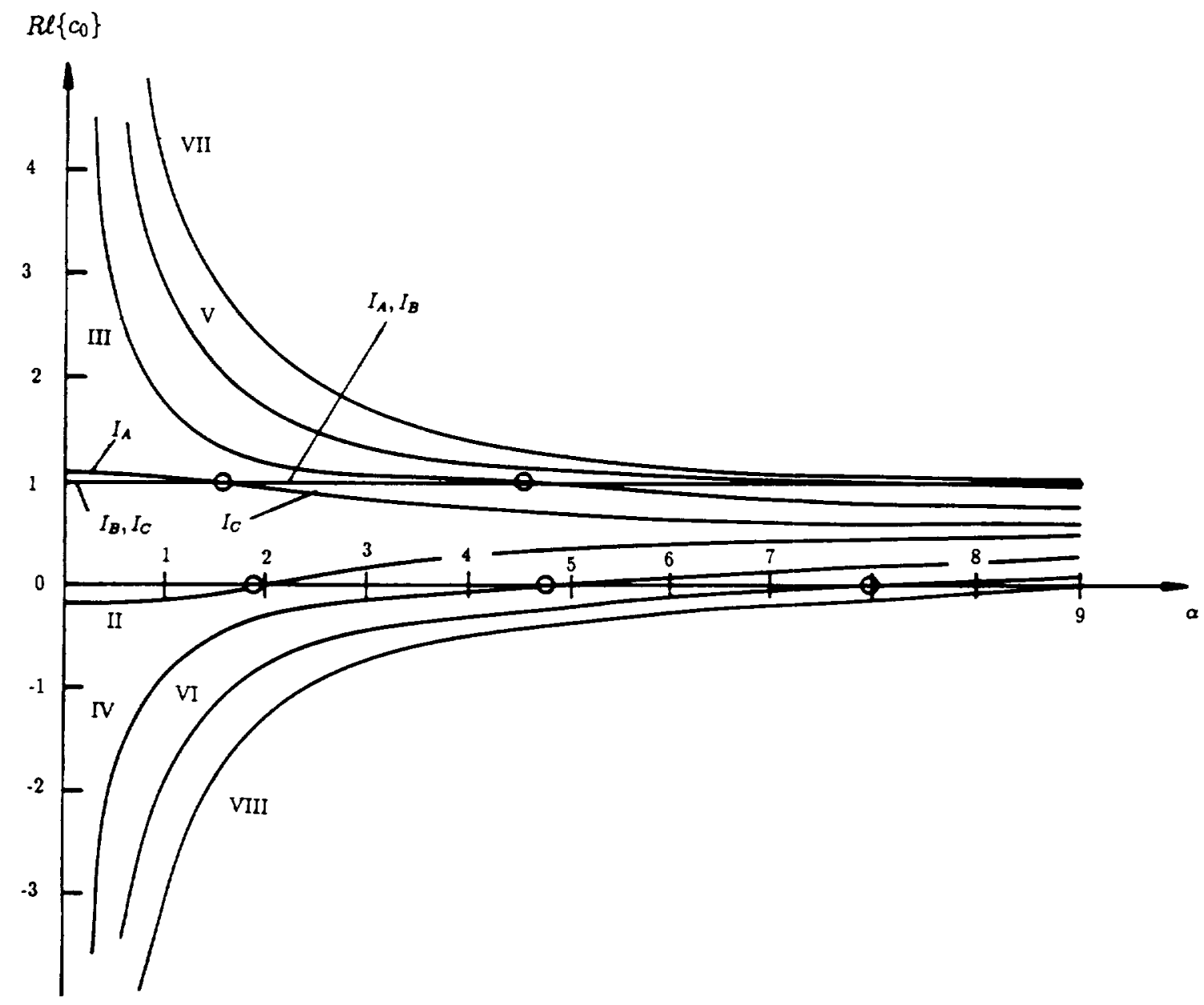

Figure 14: $R l\left\{c_{0}\right\}$ as a function of $\alpha$ for $M_{\infty}=5$, adiabatic lower wall, mode II. 


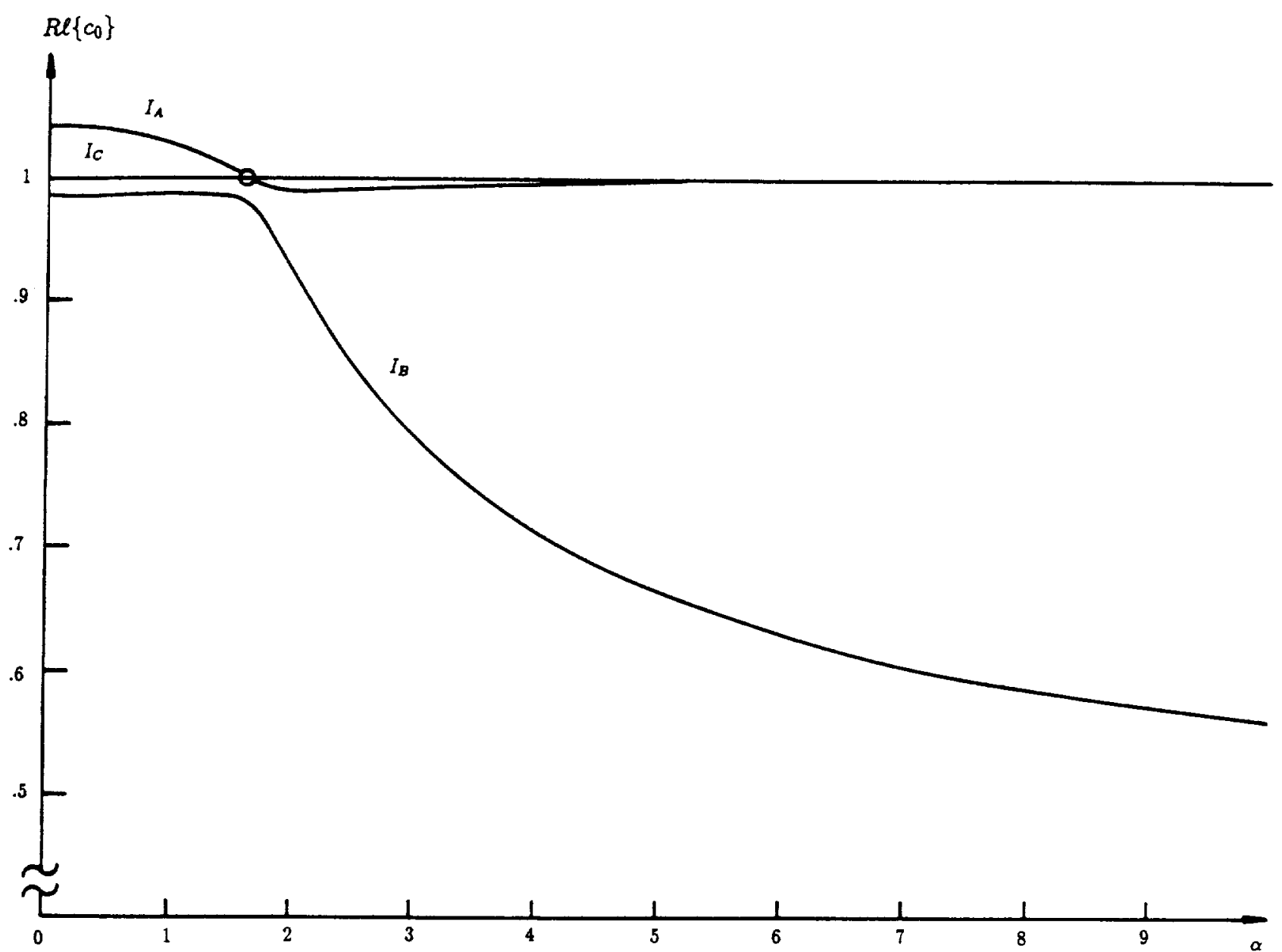

Figure 15: $R l\left\{c_{0}\right\}$ as a function of $\alpha$ for $M_{\infty}=5$, adiabatic lower wall, modes $I_{A}, I_{B}, I_{C}$. 


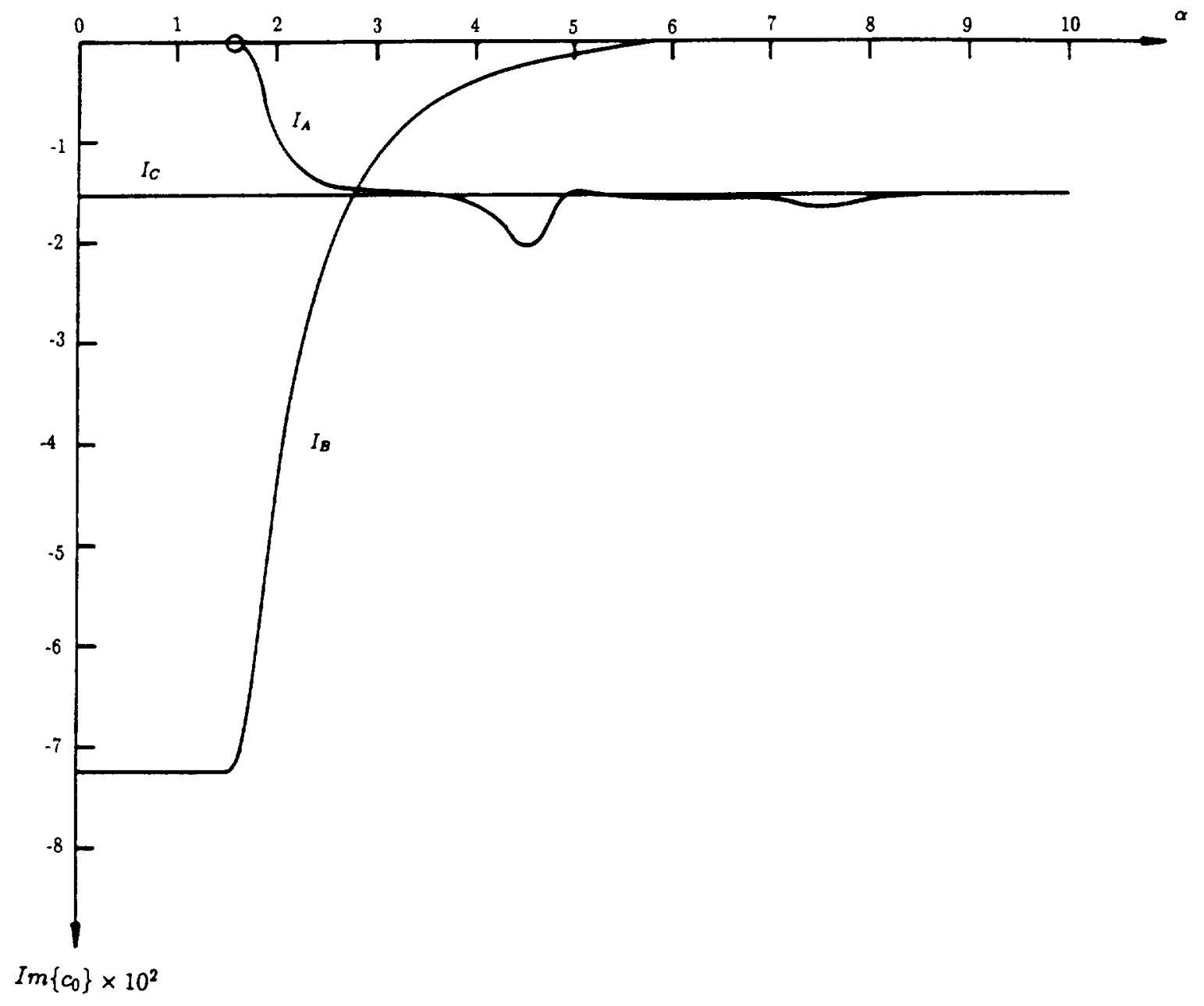

Figure 16: $\operatorname{Im}\left\{c_{0}\right\}$ as a function of $\alpha$ for $M_{\infty}=5$, adiabatic lower wall, modes $I_{A}, I_{B}, I_{C}$. 


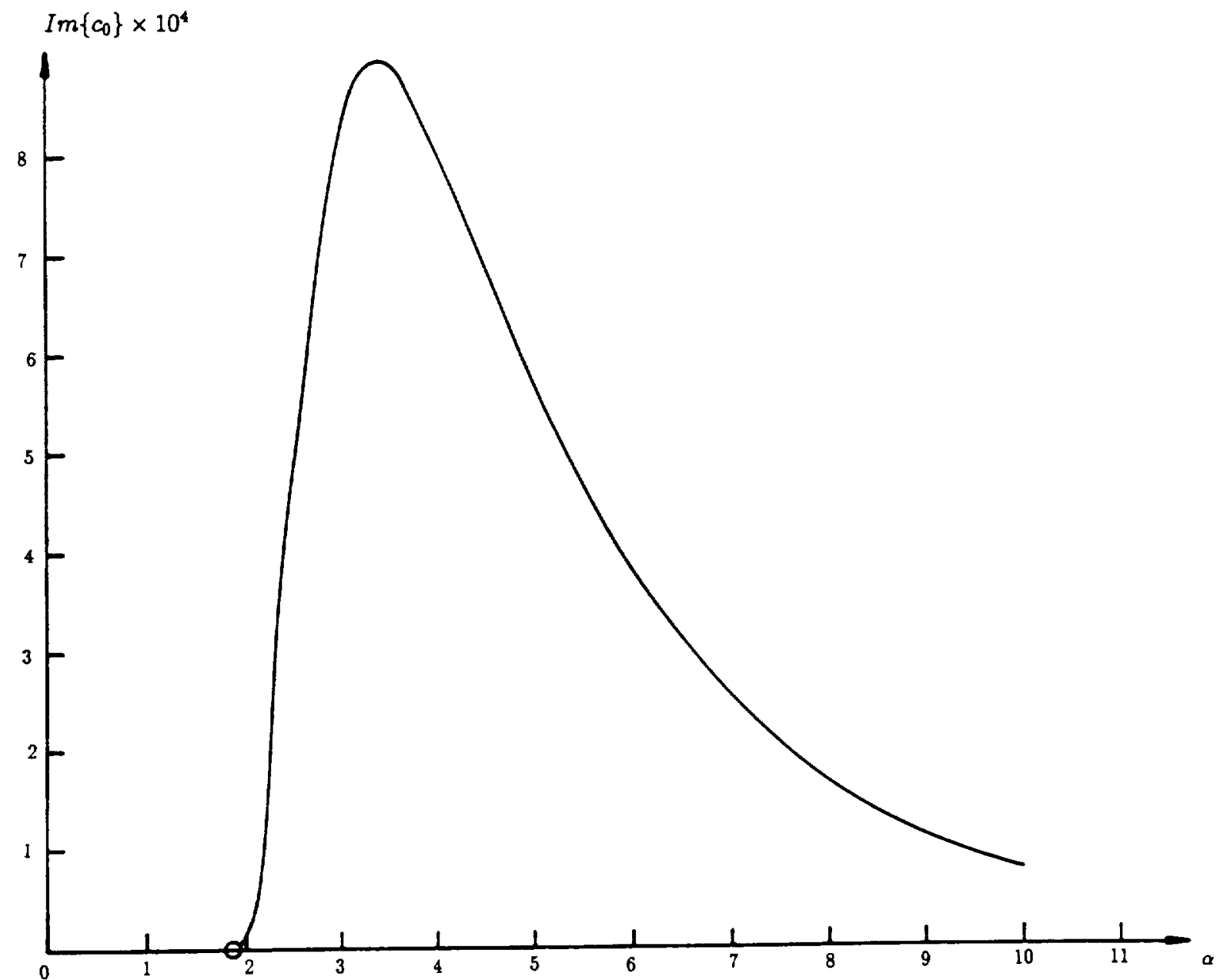

Figure 17: $\operatorname{Im}\left\{c_{0}\right\}$ as a function of $\alpha$ for $M_{\infty}=5$, adiabatic lower wall, mode II. 


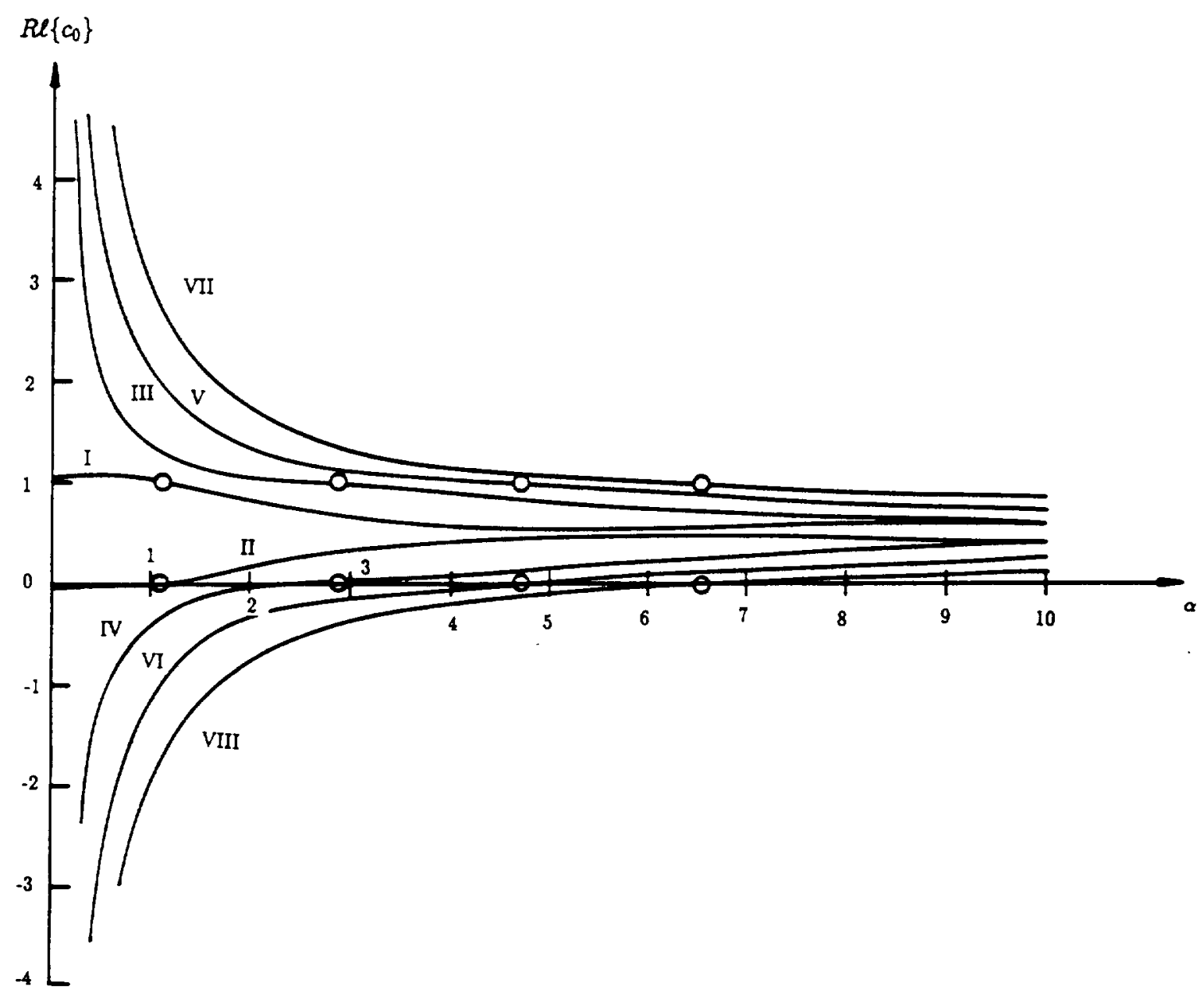

Figure 18: $R l\left\{c_{0}\right\}$ as a function of $\alpha$ for $M_{\infty}=5, T(0)=1$. 


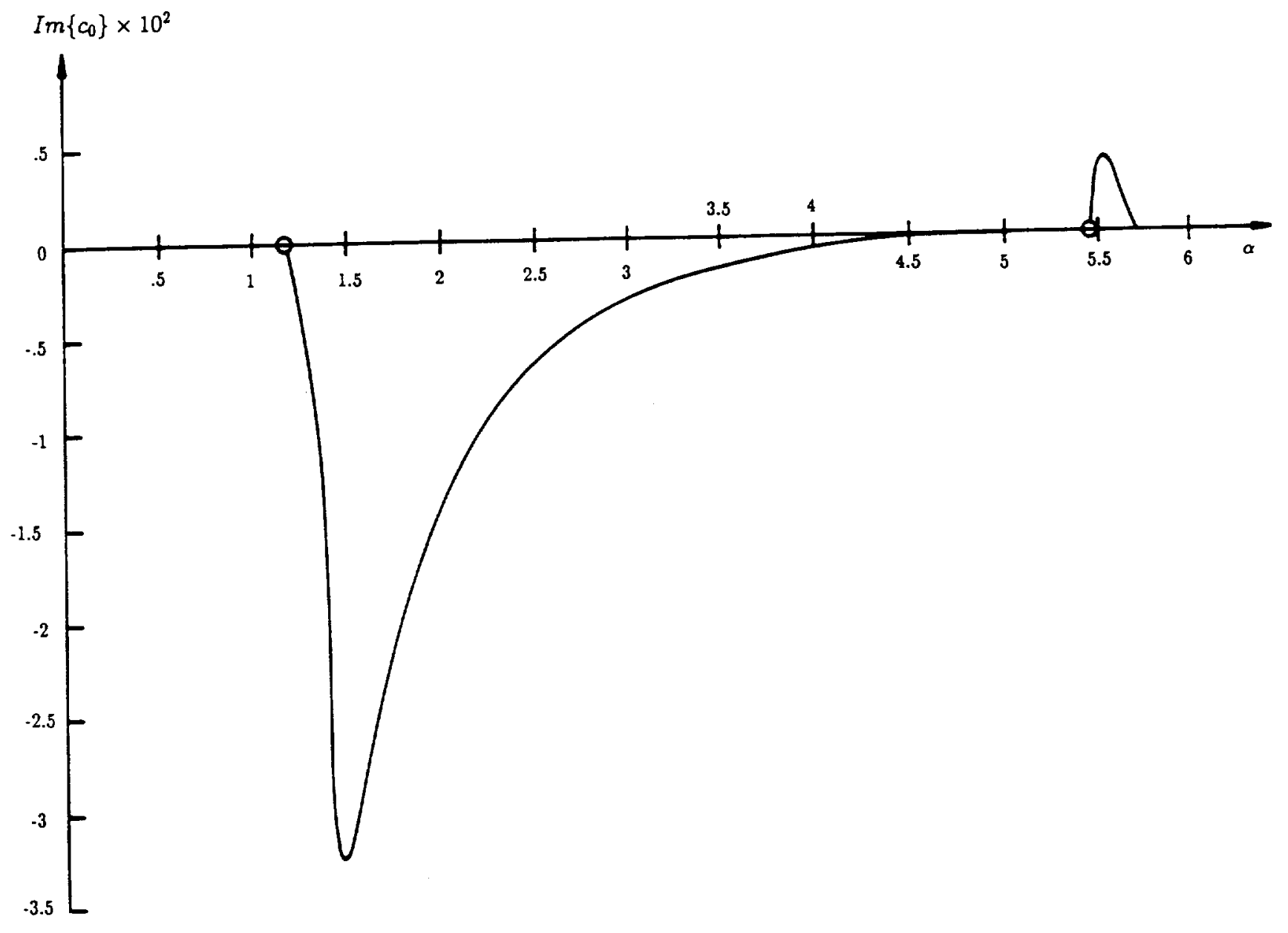

Figure 19: $\operatorname{Im}\left\{c_{0}\right\}$ as a function of $\alpha$ for $M_{\infty}=5, T(0)=1$, modes I and II. 


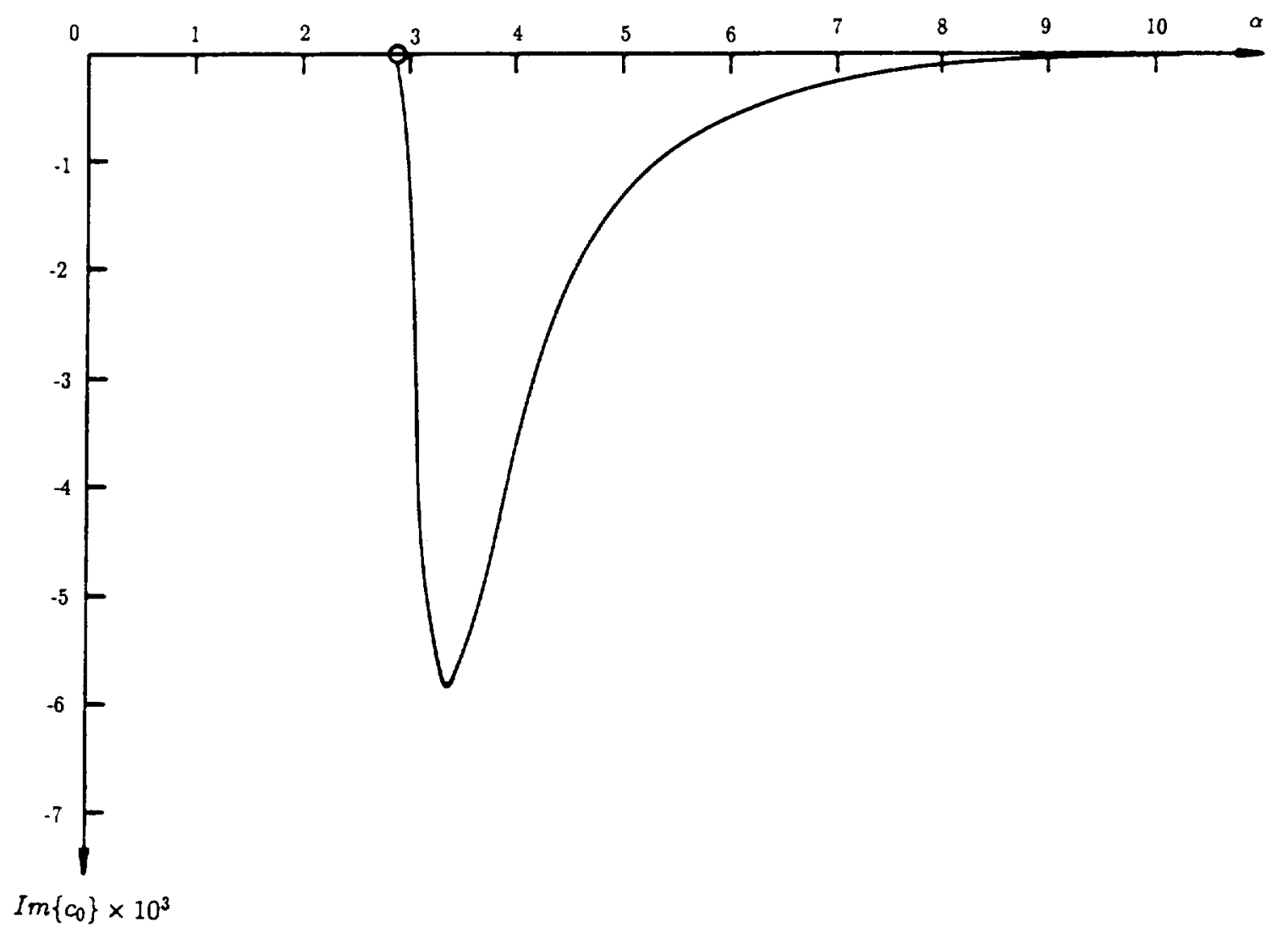

Figure 20: $\operatorname{Im}\left\{c_{0}\right\}$ as a function of $\alpha$ for $M_{\infty}=5, T(0)=1$, modes III and IV. 


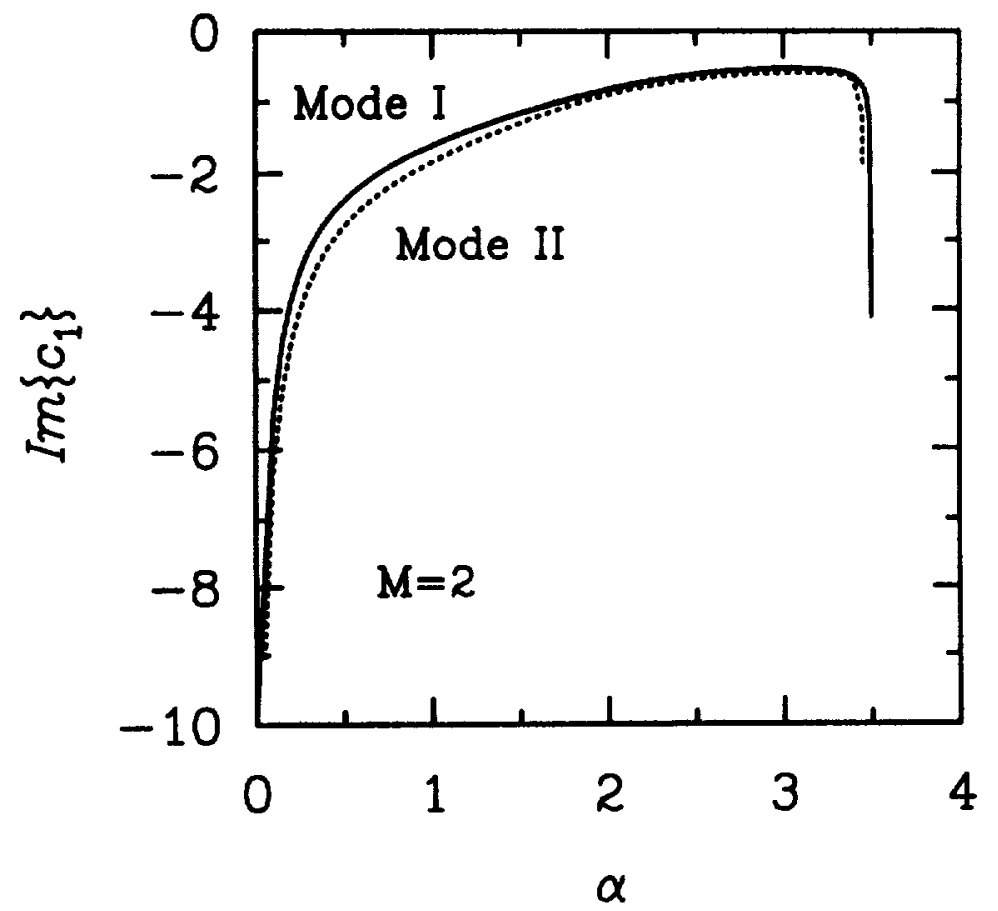

Figure 21: $\operatorname{Im}\left\{c_{1}\right\}$ as a function of $\alpha$ for $M_{\infty}=2$, adiabatic lower wall, modes I and II.



Figure 22: $\operatorname{Im}\left\{c_{1}\right\}$ as a function of $\alpha$ for $M_{\infty}=5$, adiabatic lower wall, modes I and II. 


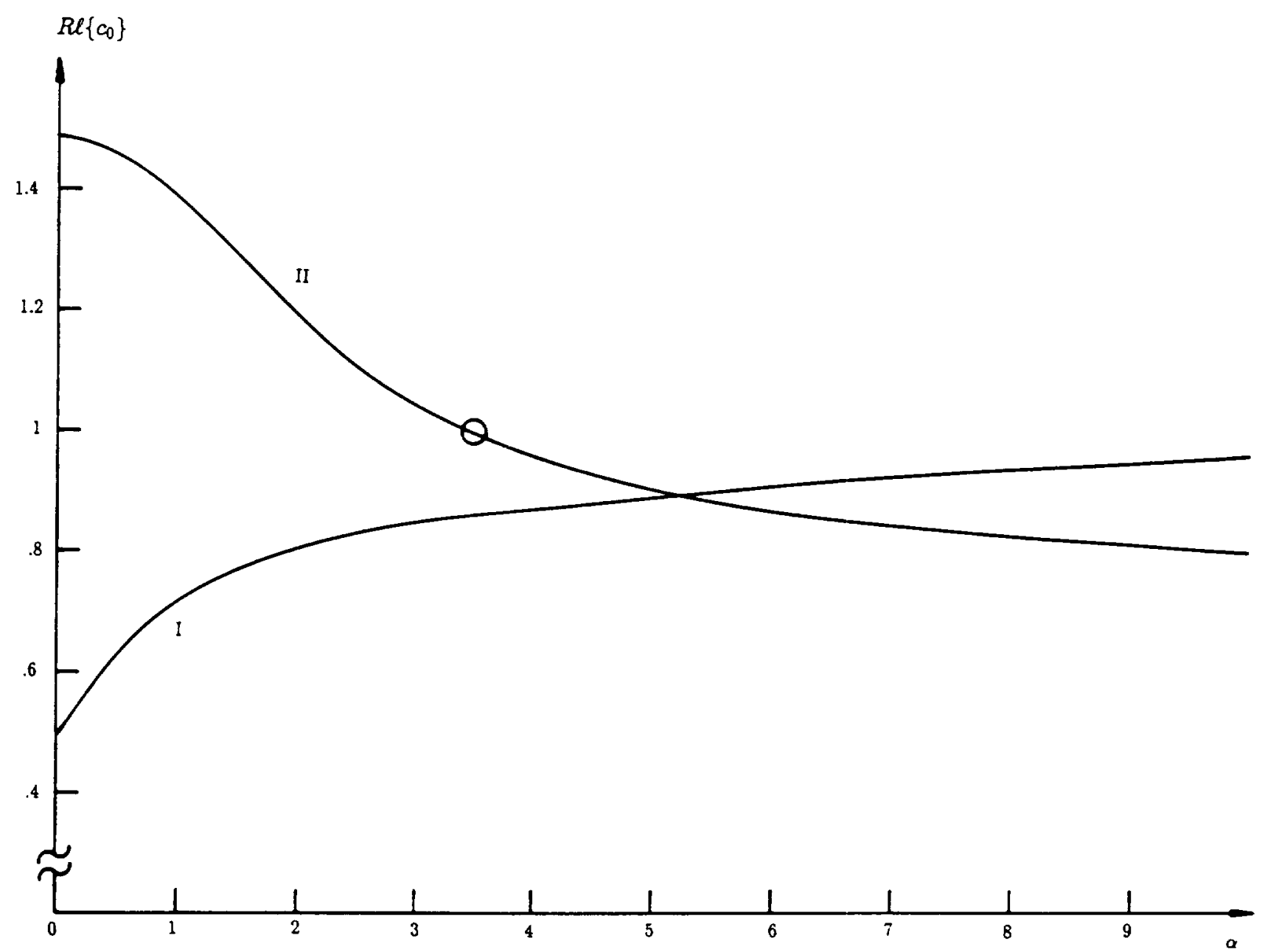

Figure 23: $R l\left\{c_{0}\right\}$ as a function of $\alpha$ for $M_{\infty}=2$, radiation boundary condition on $y=1$. 




Figure 24: $\operatorname{Im}\left\{c_{0}\right\}$ as a function of $\alpha$ for $M_{\infty}=2$, radiation boundary condition on $y=1$. 


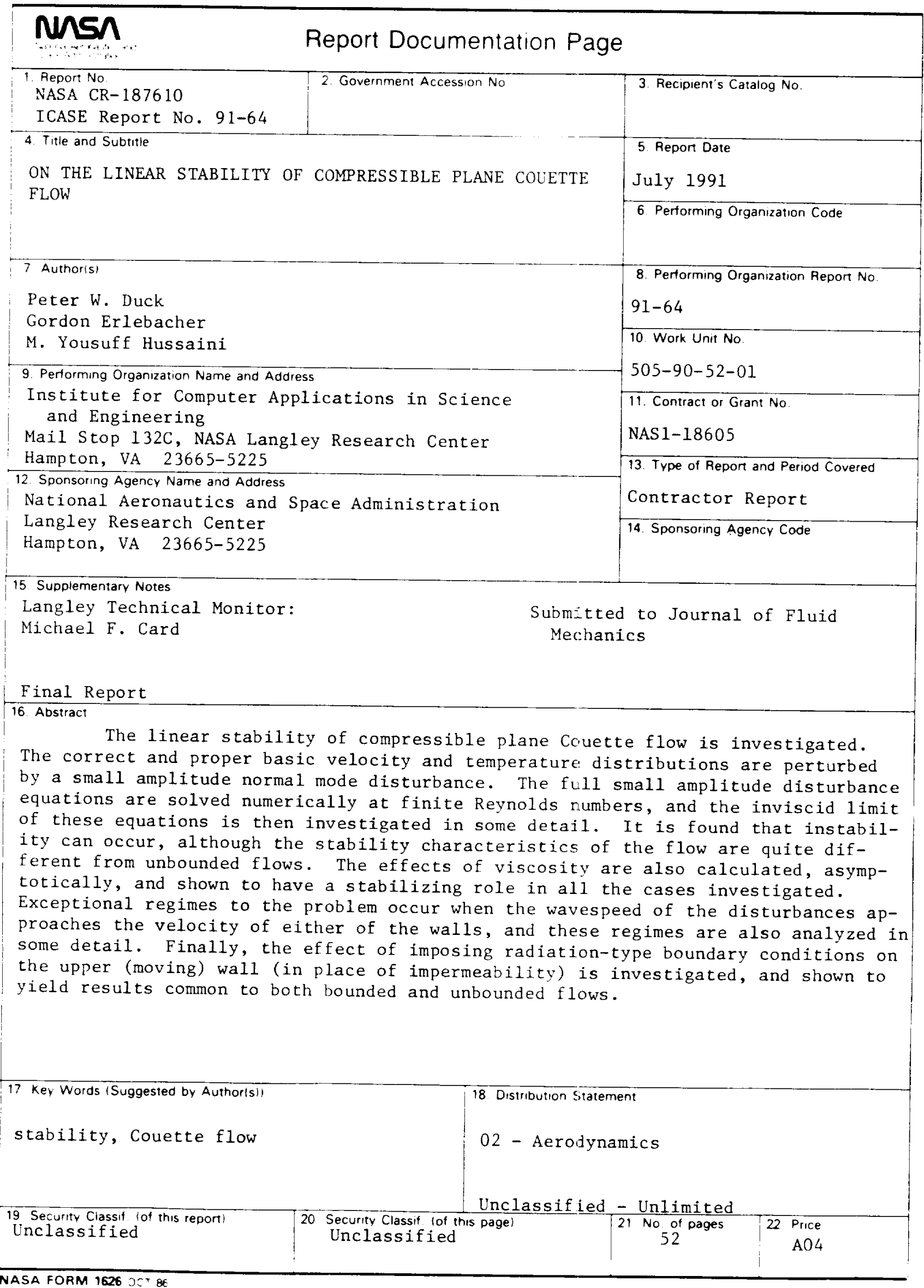


